University of New Hampshire

University of New Hampshire Scholars' Repository

Master's Theses and Capstones

Student Scholarship

Fall 2010

\title{
Investigation of strain gradients and magnitudes during microbending
}

Lijie Wang

University of New Hampshire, Durham

Follow this and additional works at: https://scholars.unh.edu/thesis

\section{Recommended Citation}

Wang, Lijie, "Investigation of strain gradients and magnitudes during microbending" (2010). Master's Theses and Capstones. 573.

https://scholars.unh.edu/thesis/573

This Thesis is brought to you for free and open access by the Student Scholarship at University of New Hampshire Scholars' Repository. It has been accepted for inclusion in Master's Theses and Capstones by an authorized administrator of University of New Hampshire Scholars' Repository. For more information, please contact Scholarly.Communication@unh.edu. 


\section{NOTE TO USERS}

This reproduction is the best copy available.

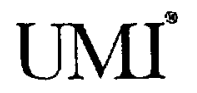




\title{
INVESTIGATION OF STRAIN GRADIENTS AND MAGNITUDES DURING MICROBENDING
}

\author{
BY \\ LIJIE WANG \\ Baccalaureate of Science, University of Tianjin, 2008
}

THESIS

Submitted to the University of New Hampshire

in Partial Fulfillment of

the Requirements for the Degree of

Master of Science

in

Mechanical Engineering

September, 2010 
UMI Number: 1486983

\section{All rights reserved}

\section{INFORMATION TO ALL USERS}

The quality of this reproduction is dependent upon the quality of the copy submitted.

In the unlikely event that the author did not send a complete manuscript and there are missing pages, these will be noted. Also, if material had to be removed, a note will indicate the deletion.

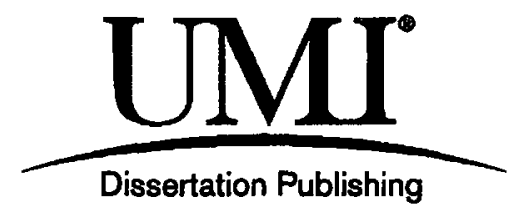

UMI 1486983

Copyright 2010 by ProQuest LLC.

All rights reserved. This edition of the work is protected against unauthorized copying under Title 17, United States Code.

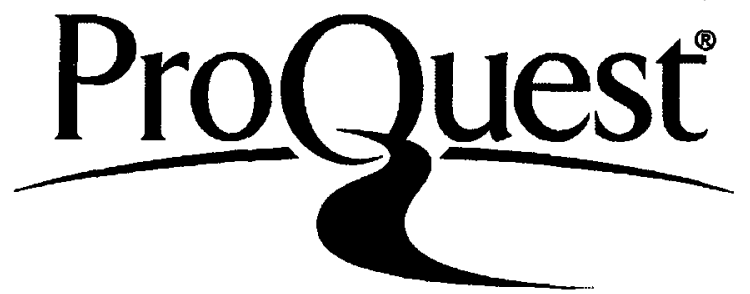

ProQuest LLC

789 East Eisenhower Parkway

P.O. Box 1346

Ann Arbor, MI 48106-1346 
This thesis has been examined and approved.
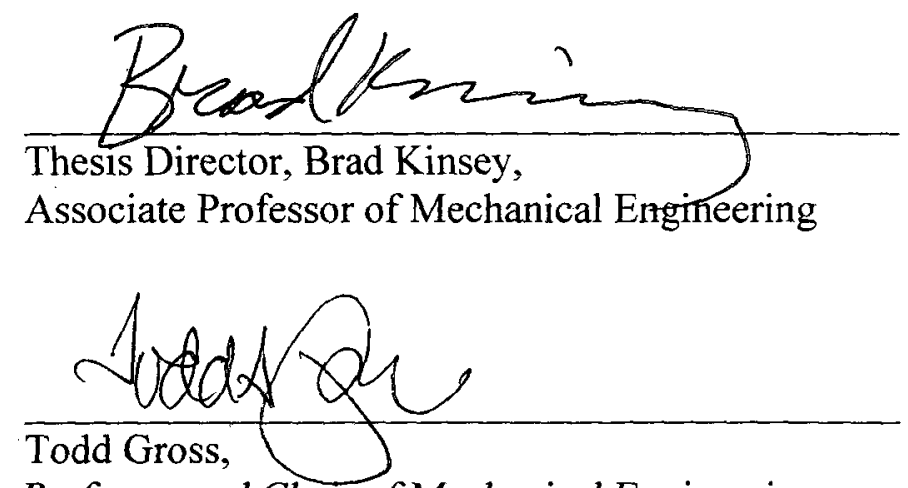

Professor and Chair of Mechanical Engineering

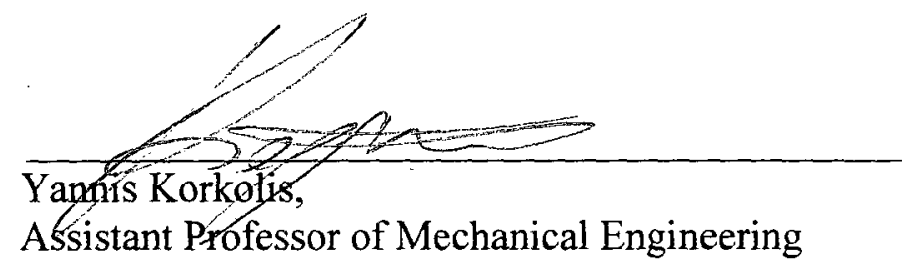

$\underset{\text { Jate }}{\text { June }} 9+h, 2010$ 


\section{ACKNOWLEDGEMENTS}

Support from NSF (CMMI-0644705) for my research is gratefully acknowledged. 


\section{TABLE OF CONTENTS}

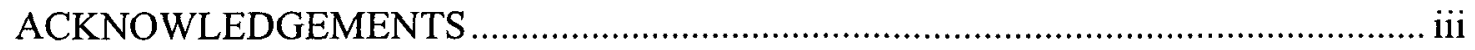

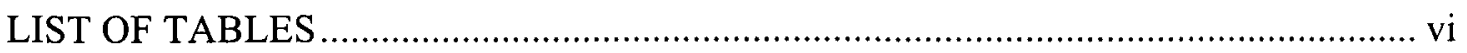

LIST OF FIGURES …………………................................................................. vii

ABSTRACT

CHAPTER PAGE

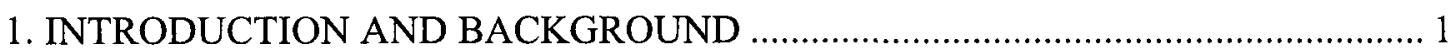

2. EXPERIMENTAL SET-UP OF MICROBENDING PROCESS ................................. 10

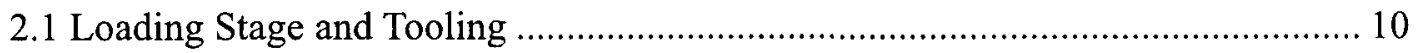

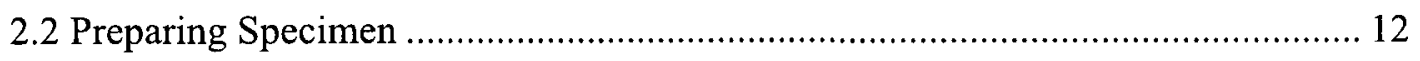

2.3 Digital Image Correlation System...................................................................... 13

2.3.1 Preparing the Specimen for DIC Analysis ....................................................... 14

2.3.2 Setting up the camera ............................................................................... 15

2.3.3 Post-processing of the DIC Data................................................................. 15

2.4 Parameter Study of DIC Parameters ………………….................................. 18

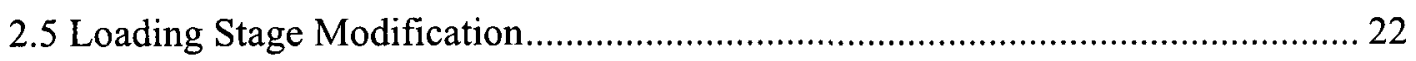

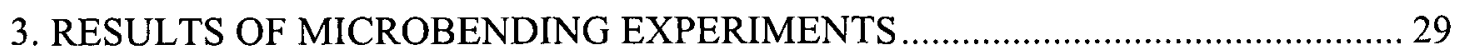

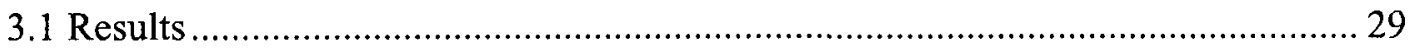

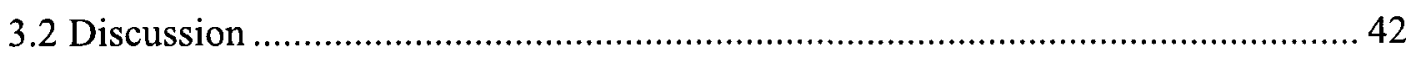

4. INVESTIGATION OF STRAIN GRADIENT HARDENING DURING

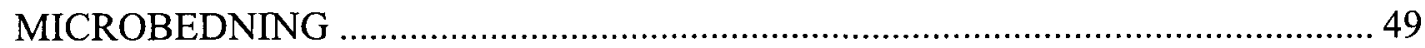




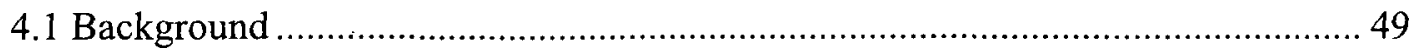

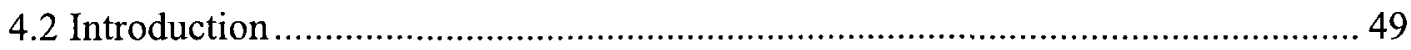

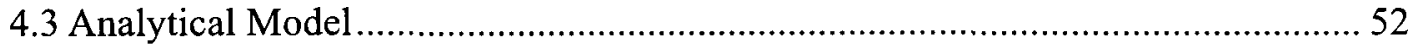

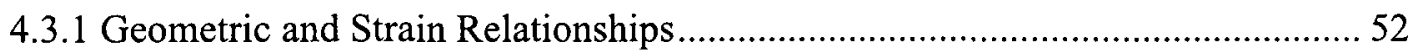

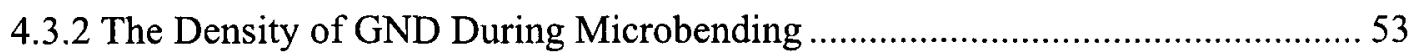

4.4 Analytical Mode Results............................................ 54

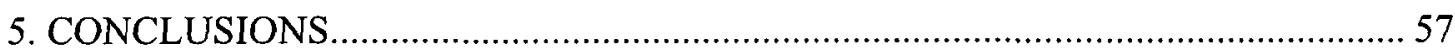

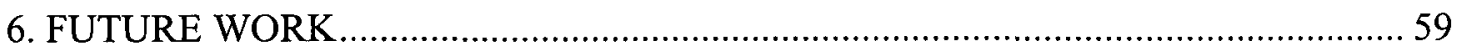

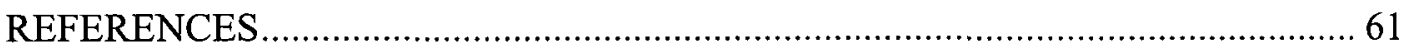

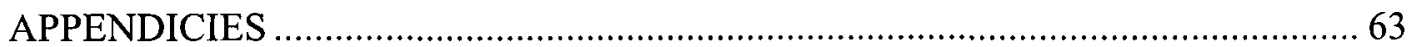

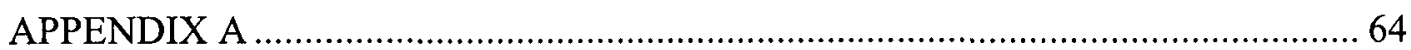

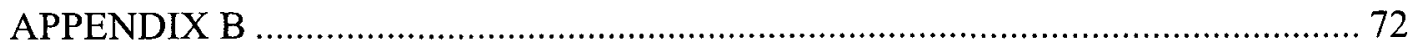




\section{LIST OF TABLES}

Table 2-1: Specimen thickness, grain size, and heat treatment information 13

Table 2-2: Average confidence (top number) and iterations (bottom number) obtained with varying subset size and step size for a) $1.625 \mathrm{~mm}$ and b) $0.5 \mathrm{~mm}$ thickness specimens. .20

Table 2-3: Strain values at various locations of the $0.5 \mathrm{~mm}$ specimen from DIC analysis results and scribed lines measurement before loading stage modification. .25 Table 2-4: Strain values at various locations of the $0.5 \mathrm{~mm}$ specimen from DIC analysis results and scribed lines measurement after loading stage modification 26

Table 3-1: Amount of neutral axis shifting for $1.625 \mathrm{~mm}, 0.5 \mathrm{~mm}$ and $0.25 \mathrm{~mm}$ thickness specimens with varying grained structures. 31 


\section{LIST OF FIGURES}

Figure 1-1: Examples of microformed parts [1]

Figure 1-2: Flow stress decreases with decreasing specimen size due to the incease in the share of surface grains [6] 3

Figure 1-3: Variation of tension and bending yield strength with varying thickness to grain size ratio $(\varphi)[7]$

Figure 1-4: Average hardness distribution at the bend area through the thickness of a)

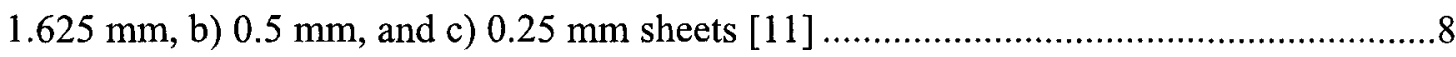

Figure 1-5: Average hardness distribution at the bend area through the thickness of a) 20

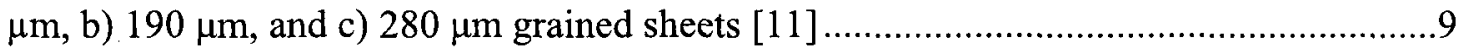

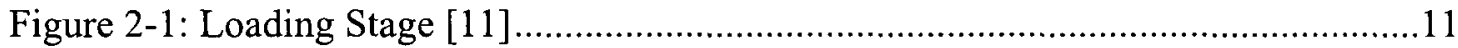

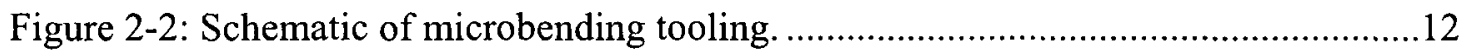

Figure 2-3: Pattern applied by airbrush on the surface of a specimen.........................15

Figure 2-4: Set-up of camera, lens, and loading stage on a steel board........................17

Figure 2-5: Full field, strain distribution data from DIC analysis ................................17

Figure 2-6: Schemetic of subset and step sizes for DIC analysis ................................18

Figure 2-7: The strain distribution data with the varied subset size and specific step for a)

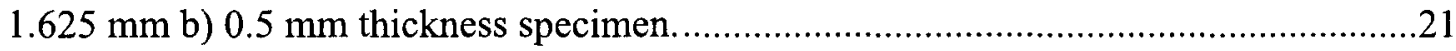

Figure 2-8: The strain distribution data with the varied step and specific subset size for a)

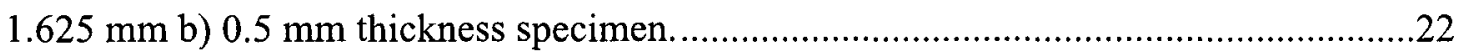

Figure 2-9: Specimen with scribed lines (a) before bending and (b) after bending. ........25

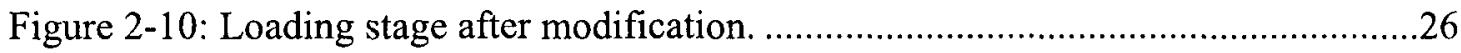


Figure 2-11: Schematic of the neutral axis location after bending.. .28

Figure 3-1: Average strain distribution at the bend area directly under the punch through the thickness of a) $1.625 \mathrm{~mm}, \mathrm{~b}) 0.5 \mathrm{~mm}$ and c) $0.25 \mathrm{~mm}$ sheets. 31

Figure 3-2: Average absolute strain value distribution directly under the punch through the thickness for a) $1.625 \mathrm{~mm}$, b) $0.5 \mathrm{~mm}$ and c) $0.25 \mathrm{~mm}$ sheets with varying grain structures. .33

Figure 3-3: Average absolute strain distribution through the thickness for the a) fine, b) medium and c) coarse grained structures........

Figure 3-5: Strain gradient slope changes for $1.625 \mathrm{~mm}, 0.5 \mathrm{~mm}$ and $0.25 \mathrm{~mm}$ thickness specimen with varying grain structure .37

Figure 3-6: No shape change with miniturization .38

Figure 3-7: Normalized peak bending force versus specimen size curves for fine, medium and coarse grained structures 40

Figure 3-8: Normalized peak bending force versus specimen size curves for fine, medium and coarse grained structures .41

Figure 3-9: The strain distribution results obtained from DIC analysis were compared to the previous results obtained by hardness measurement in Parasiz et al. [11] for 1.625 $\mathrm{mm}$ and $0.25 \mathrm{~mm}$ thickness specimens with various grained structures. .44 Figure 3-10: The strain distribution results obtained from DIC analysis were compared to the previous results obtained by hardness measurement in Parasiz et al. [11] for specimens with fine and coarse grained structures. .45

Figure 3-11: Strain distribution data for a) $1.625 \mathrm{~mm}$ thickness specimen with fine grain structure, b) $0.5 \mathrm{~mm}$ thickness specimen with coarse grain structure. .46 
Figure 3-12: Strain distribution data for a) $1.625 \mathrm{~mm}$ thickness specimen with fine grain structure, b) $0.5 \mathrm{~mm}$ thickness specimen with coarse grain structure. 48

Figure 3-13: Maximum strain on tension surface for various specimen thicknesses and grain structures. .48

Figure 4-1: Dependence of strength on specimen size during microtorsion of thin copper wires [20]. .51

Figure 4-2: Geometric parameters for pure bending. .52

Figure 4-3: Dislocation density increase versus specimen thickness ( $\mathrm{t}$ ) for $\mathrm{CuZn30}$ and a radius of curvature of $1.5 \mathrm{t}$. .54

Figure 4-4: Normalized punch force for 3-point bending experiments with $\mathrm{CuZn} 30$ and fine grain structure. .55

Figure 4-5: Data from Stolken and Evans [14] with an assumed length scale of $5.3 \mu \mathrm{m} .56$ Figure A-1: Selecting an area of interest. .66

Figure A-2: Image analysis result window. .69

Figure A-3: Contour plot of strain distribution data. .71 


\section{ABSTRACT \\ INVESTIGATION of STRAIN GRADIENTS AND MAGNITUDES \\ DURING MICROBENDING}

by

\section{Lijie Wang}

University of New Hampshire, September, 2010

Sheet metal forming of parts with microscale dimensions is gaining importance due to the current trend towards miniaturization, especially in the electronics industry. In microforming although the process dimensions are scaled down, the polycrystalline material stays the same (e.g., the grain size remains constant). When the specimen feature size approaches the grain size, the properties of individual grains begin to affect the overall deformation behavior. This results in inhomogeneous deformation and increased data scatter of the process parameters. In this research, the influence of the specimen size and the grain size on the distribution of plastic deformation through the thickness during 3-point microbending operations is investigated via digital image correlation. Results showed that with miniaturization, a decrease in the strain gradient existed. In addition, an analytical model to predict the dislocation density increases, and thus strain gradient hardening, during microbending is presented. The results from this analytical model matched the experimental results and previous research in terms of the feature size where modest and significant strain gradient hardening was observed. 


\section{CHAPTER 1}

\section{INTRODUCTION AND BACKGROUND}

Nowadays hundreds of microscale devices have been employed in a wide array of applications including consumer electronics, biomedical technology, energy devices, etc. With minimization, microforming is a desirable process for bulk production of microscale products due to its low cost, high production rate and superior material utilization. Examples of microformed parts are shown in Fig. 1-1. However, the development of such metal forming processes for microscale mechanical components is difficult due to size effects, i.e., variations in the material, process parameters and deformation with miniaturization.

With miniaturization, the process and workpiece dimensions are scaled down while the individual grain size remains the same. Thus, the volume percentage occupied by an individual grain in the material of workpiece increases [2]. When the grain size to specimen size ratio increases significantly, the properties of individual grains begin to affect the overall deformation behavior. The result is inhomogeneous deformation and shape anomalies in the final product and scattering in the process parameters due to the anisotropic nature of individual grains [3, 4]. 


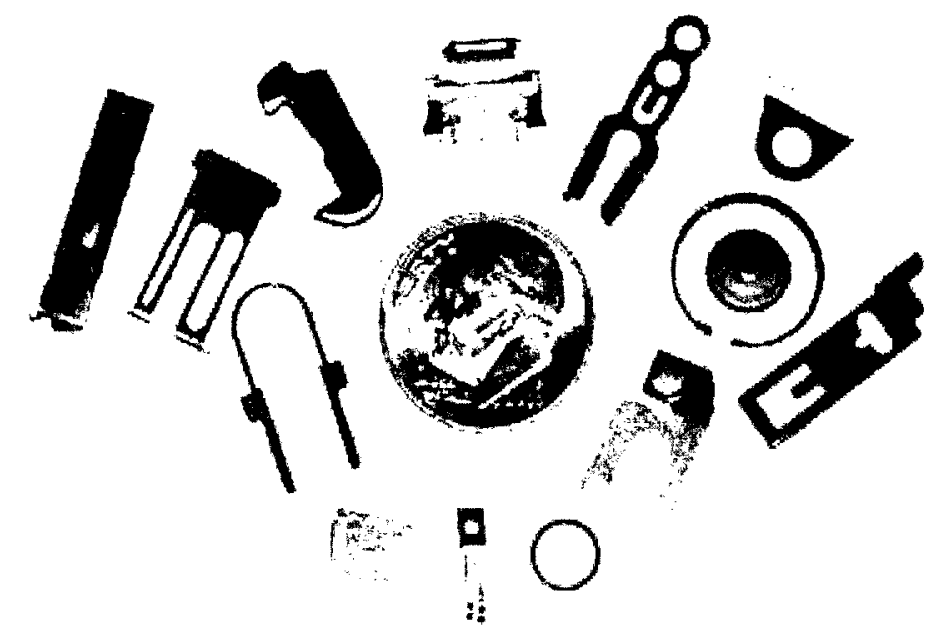

Figure 1-1: Examples of microformed parts [1].

Furthermore, during deformation the surface grains in a metallic material are constraint less than inner grains. Thus, they deform more easily and have lower flow stresses than the inner grains. As the specimen size decreases with the grain size remaining constant, the share of the surface grains in the total volume will increase; thus, the overall strength of the materials will decrease [5]. For example, Geiger et al. [6] investigated size effects by conducting tensile tests on cylindrical CuZn 15 samples with a diameter of 0.2 to $2 \mathrm{~mm}$ (where $\lambda=1$ corresponded to a diameter of $2 \mathrm{~mm}$ ) and a constant grain size of $79 \mu \mathrm{m}$. It was observed that the flow stress decreased with decreasing specimen size due to the increase in the share of surface grains, see Fig. 1-2. Also, data scatter increased with miniaturization due to the significant effect of individual grains on the deformation. 


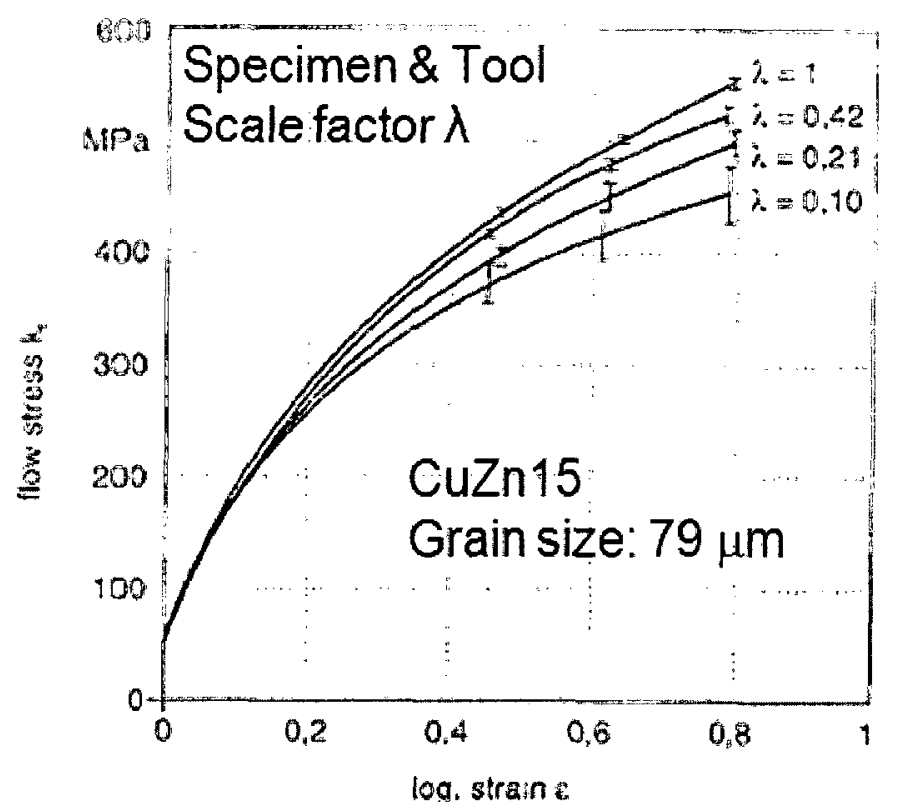

Figure 1-2: Flow stress decreases with decreasing specimen size due to the increase in the share of surface grains [6].

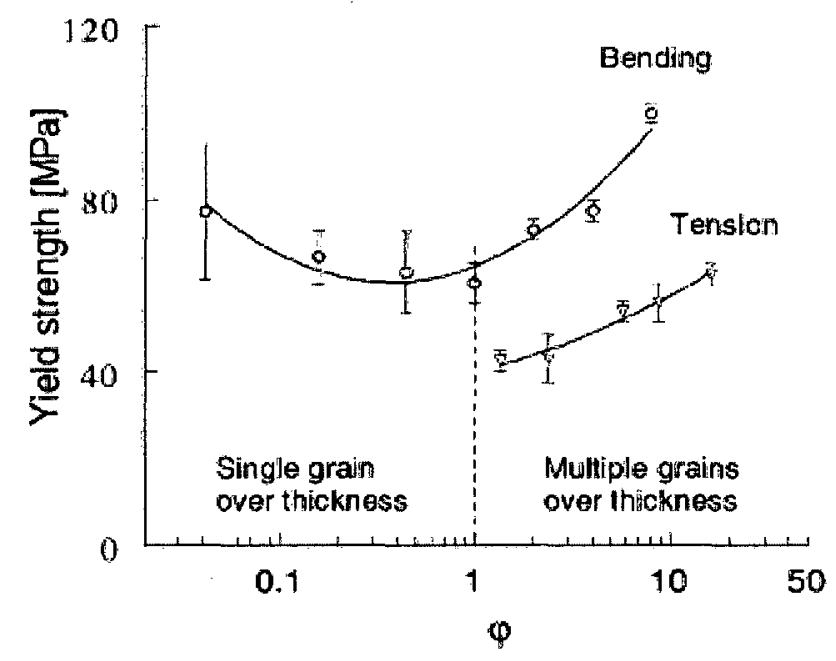

Figure 1-3: Variation of tension and bending yield strength with varying thickness to grain size ratio $(\varphi)[7]$ 
In addition, Raulea et al. [7] performed uniaxial tensile and bending tests to study size effects for Aluminum 99.0 - 99.5\% (2S Half-Hard DIN 1747 Euro-norm) and observed similar results with respect to yield stress. But when there was less than one grain through the thickness obtained by measuring planar grain size and dividing into thickness, the yield stress in bending experiments increased with increasing grain size. This is again due to the effect of individual grain in the material, see Fig. 1-3.

Bending is a flexible metal forming process, by which many different shapes can be produced using different die and punch sets. During bending, the material on the surface of the workpiece away from the punch is stretched due to tensile loading, while the material on the other surface nearest the punch is compressed by compressive loading. The engineering strain increases linearly from the neutral axis [8], i.e., where strain is zero, to the surface region of the workpiece. The maximum absolute value of strain occurs on both the tension and compression sides of the sheet. Similarly, the stress increases gradually from the neutral axis to the maximum absolute value at the surfaces. The neutral axis is initially in the center of the metal sheet but shifts towards the compression side during bending [9]. Initially, the metal experiences only elastic deformation. When the metal is stressed above its elastic limit, it is plastically deformed. The plastic deformation increases as the degree of bending increases, and the plastic deformation penetrates from the surface into the inner region [10]. Thus, analyzing the stress and strain distribution during a bending process can give a clear understanding of this inhomogeneous deformation. 
Past microbending experiments were conducted with CuZn30 material [11]. For these experiments, the thickness was varied to $0.25 \mathrm{~mm}, 0.5 \mathrm{~mm}$ and $1.625 \mathrm{~mm}$; the process geometries (both specimen and tooling) were functions of the thickness and adjusted based on the law of similarity so a consistent strain is predicted and results from various geometries can be compared by normalizing parameters; and the grain size was manipulated through heat treatment to vary the number of grains through the thickness. Knoop microhardness investigations were performed to assess deformation size effects with miniaturization. Figure 1-4 shows that as the thickness decreases (e.g. from 1.625 $\mathrm{mm}$ to $0.25 \mathrm{~mm}$ ), although the specimens underwent the same scaled microbending processes, variations in the hardness or deformation distribution occur through the thickness. In Fig. 1-4 a), for the $1.625 \mathrm{~mm}$ specimens, the hardness is consistent for the various grain structures. For $0.5 \mathrm{~mm}$ and $0.25 \mathrm{~mm}$ specimens in Figs. 1-4 b) and c) respectively, the penetration of plastic deformation occurs from the surface regions to the inner region causing higher hardness values in the center of the specimen. Note that the zero location in Fig. 1-4 is the center of the sheet with positive location values corresponding to the tension side of the bending process and negative values being the compression side and that the initial hardness values were subtracted. Each plot is an average hardness increase distribution of two specimens in the bend area in a $4 \mathrm{t}$ region around where the punch tip contacts the specimen. Measurements were obtained with a $10 \mathrm{~g}$ load and are spaced approximately $50 \mu \mathrm{m}$ apart for the $0.25 \mathrm{~mm}$ and $0.5 \mathrm{~mm}$ thick specimens and $75 \mu \mathrm{m}$ apart for the $1.625 \mathrm{~mm}$ thick specimens. Similar spacings were used to the specimen's surfaces as well. The spacing of indents assured that adjacent measurements were not affected by work hardening of the material around the indents. 
See Parasiz et al. [11] for further details. But the fact that processes geometries were scaled does not guarantee strains will be equal, so limitations exist in this methodology.

In Fig. 1-5, to compare the deformation of the fine, medium and coarse grained specimens for the different thicknesses, the locations through the thickness were normalized by dividing by half of the specimen's thickness. The hardness distributions for the three different specimen sizes were plotted together for a given grain size. For Fig. 1-5 a) with a grain size of $20 \mu \mathrm{m}$, the deformation distribution through the thickness is not affected significantly by miniaturization. However, in Figs 1-5 b) and c) for the 190 $\mu \mathrm{m}$ and $280 \mu \mathrm{m}$ specimens respectively, the thinner specimens have higher hardness values in the center of the specimen. This shows the penetration of the plastic deformation to the inner regions during microbending of the coarse grained specimens as a result of miniaturization.

While interesting results were obtained in these hardness studies, there are several disadvantages to this technique, e.g., it is time-consuming to perform the analyses, contact-measurements may damage the surface of specimen, and continuous data is not possible due to spacing between hardness measurements. In order to improve the assessment of deformation during 3-point microbending operations, full-field visual strain measurements from Digital Image Correlation (DIC) are used in this research to investigate the influence of the workpiece size and the number of grains through the cross-section on the distribution of plastic deformation through the thickness of the metal sheets. In Chapter 2, the experimental set-up is presented including a parametric study of 
the DIC parameters. In Chapter 3, strain distribution and force-displacement data is presented demonstrating deformation size effects. The strain distribution data is of particular interest to understand these deformation size effects and is essential for accurate determination of Strain Gradient Hardening (SGH). In Chapter 4, an analytical model is presented to calculate the Geometrically Necessary Dislocation (GND) density and SGH during a bending process. This formulation was used for the experiment case to show the effect of initial sheet thickness on Geometrically Necessary Dislocation (GND) density. The results are in reasonable agreement with past research conducted by Parasiz et al. [11] and by Stolken and Evans [12] for modest and significant increases in SGH respectively. Finally, in Chapter 5 and 6, conclusions and future direction for the research are discussed.

a)

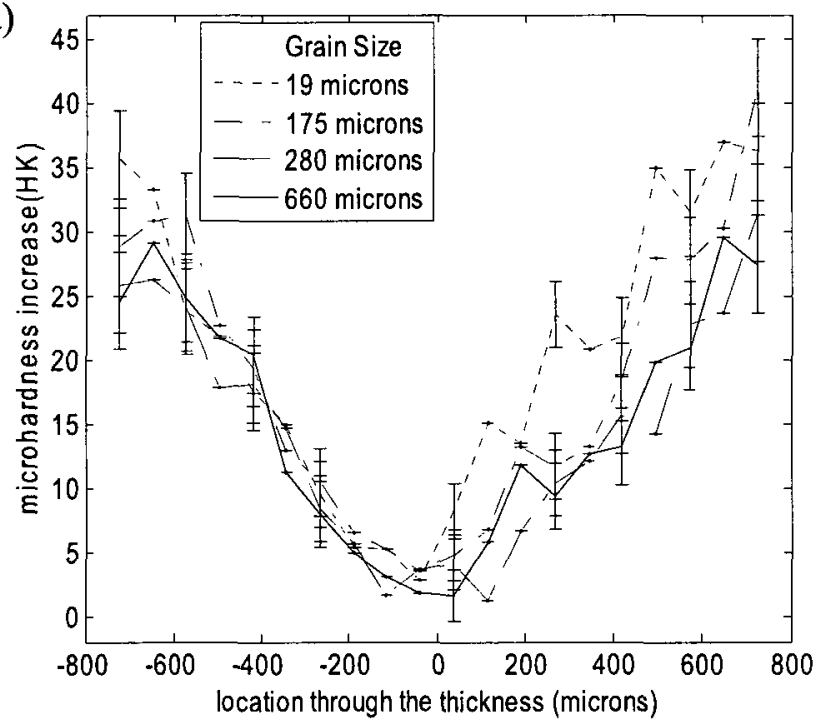


b)

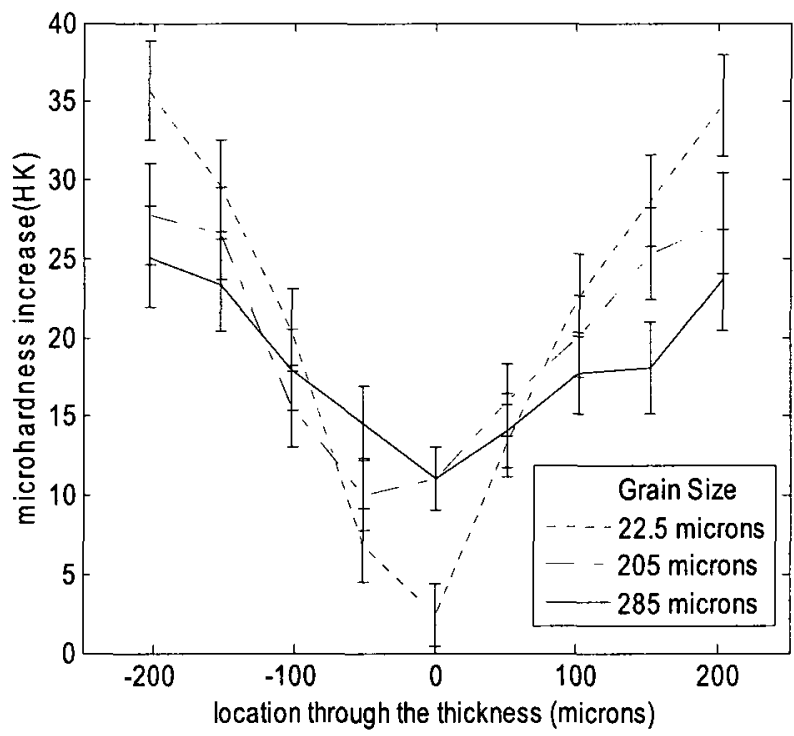

c)

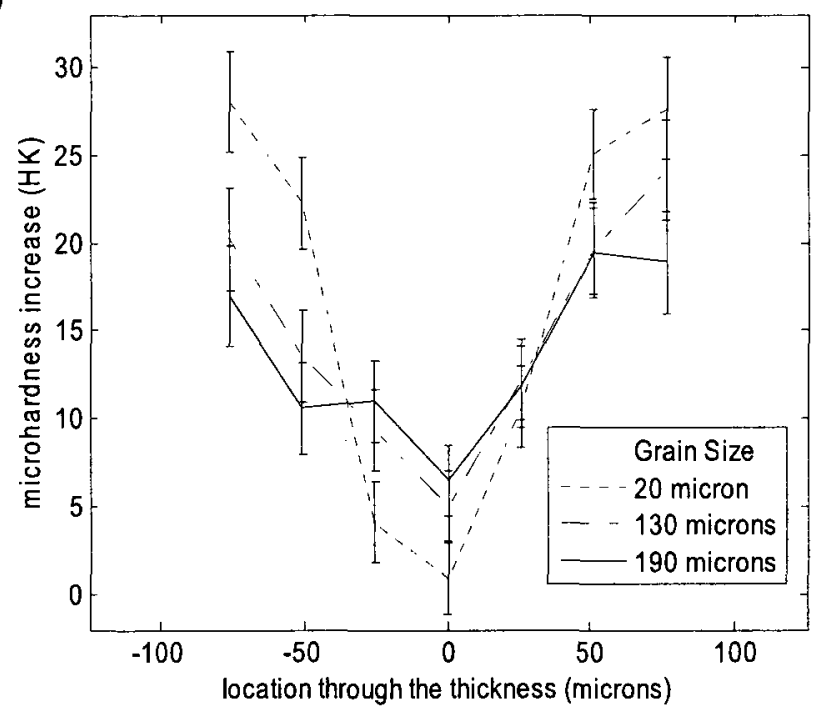

Figure 1-4: Average hardness distribution at the bend area through the thickness of a) $1.625 \mathrm{~mm}$, b) $0.5 \mathrm{~mm}$, and c) $0.25 \mathrm{~mm}$ sheets [11]. 
a)

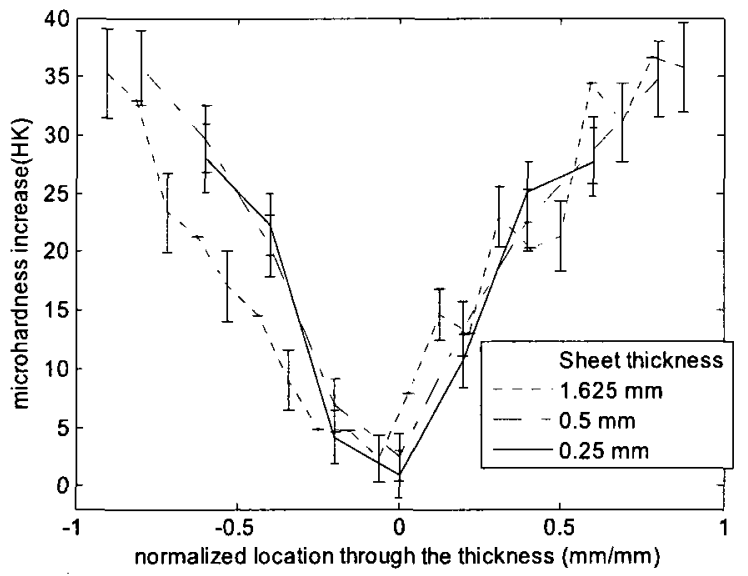

b)

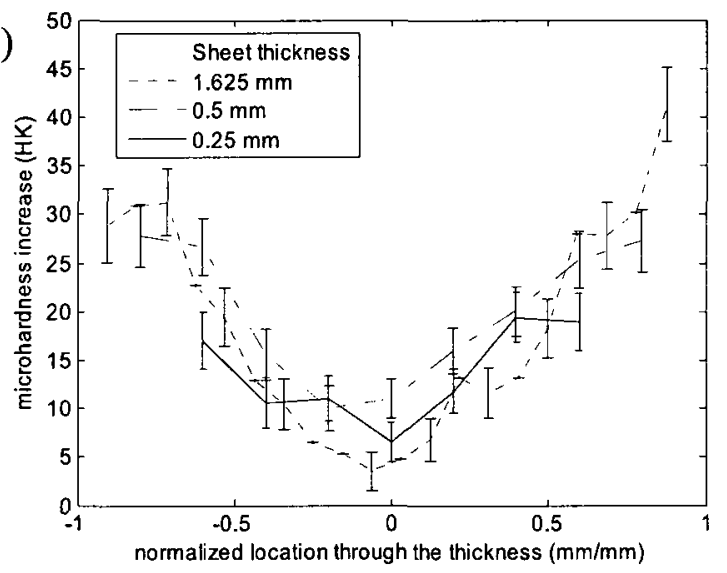

c)

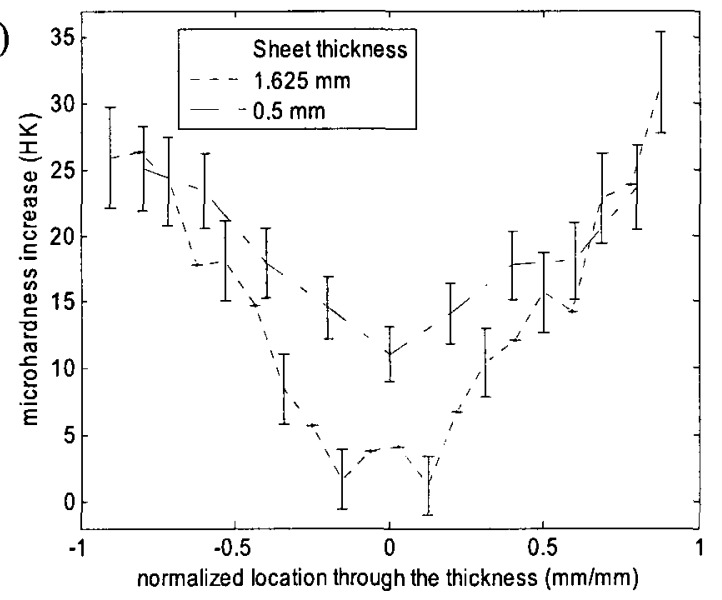

Figure 1-5: Average hardness distribution at the bend area through the thickness of a) 20 $\mu \mathrm{m}$, b) $190 \mu \mathrm{m}$, and c) $280 \mu \mathrm{m}$ grained sheets [11]. 


\section{EXPERIMENT SET-UP OF MICROBENDING PROCESS}

As indicated in Figs. 1-4 and 1-5, past microbending experiments have been conducted with $\mathrm{CuZn} 30$ material which is the typical material of interests for electronic study. For these experiments, the thickness was varied from 0.25 to $1.625 \mathrm{~mm}$; the process geometries (both specimen and tooling) were adjusted based on the law of similarity so a consistent strain is predicted; and the grain size was manipulated through heat treatment to vary the number of grains through the thickness. Microhardness investigations were performed to assess deformation size effects with miniaturization. However, understanding the strain distribution through the thickness during microbending is also of interest. Thus, an experimental set-up which includes Digital Image Correlation (DIC) was implemented.

\subsection{Loading stage and Tooling}

The microbending experiments were performed on a $4448 \mathrm{~N}$ (1000 lb) SEM loading stage manufactured by Ernest F. Fullam Inc., NY, see Fig 2-1. The bending tooling was designed according to the theory of similarity. Thus, the same deformation (i.e., strain) is imposed on the specimens by the tooling for each size. Both of the ratios of the punch radius $R_{p}$ and die radius $R_{d}$ to the thickness of sheets are 1.5 , and the die gap has a $2 t$ clearance from both sides of the punch. Thus, the total width of the die opening $L_{d}$ is $7 t$. 
See Fig. 2-2 for a schematic of the tooling. The displacement was measured using a Vishay Micro-Measurements Linear Displacement Sensor HS25 with a fully active 350ohm strain gauge bridge to sense displacement. The bending force was measured using a 111.2 N (25 lb) Sensotec precision miniature load cell model 31 for the $0.5 \mathrm{~mm}$ and 0.25 mm thickness specimens and a $2224 \mathrm{~N}(500 \mathrm{lb})$ load cell for the $1.625 \mathrm{~mm}$ cases. The accuracy of the load cells is $\pm 0.13 \%$ of the full scale.

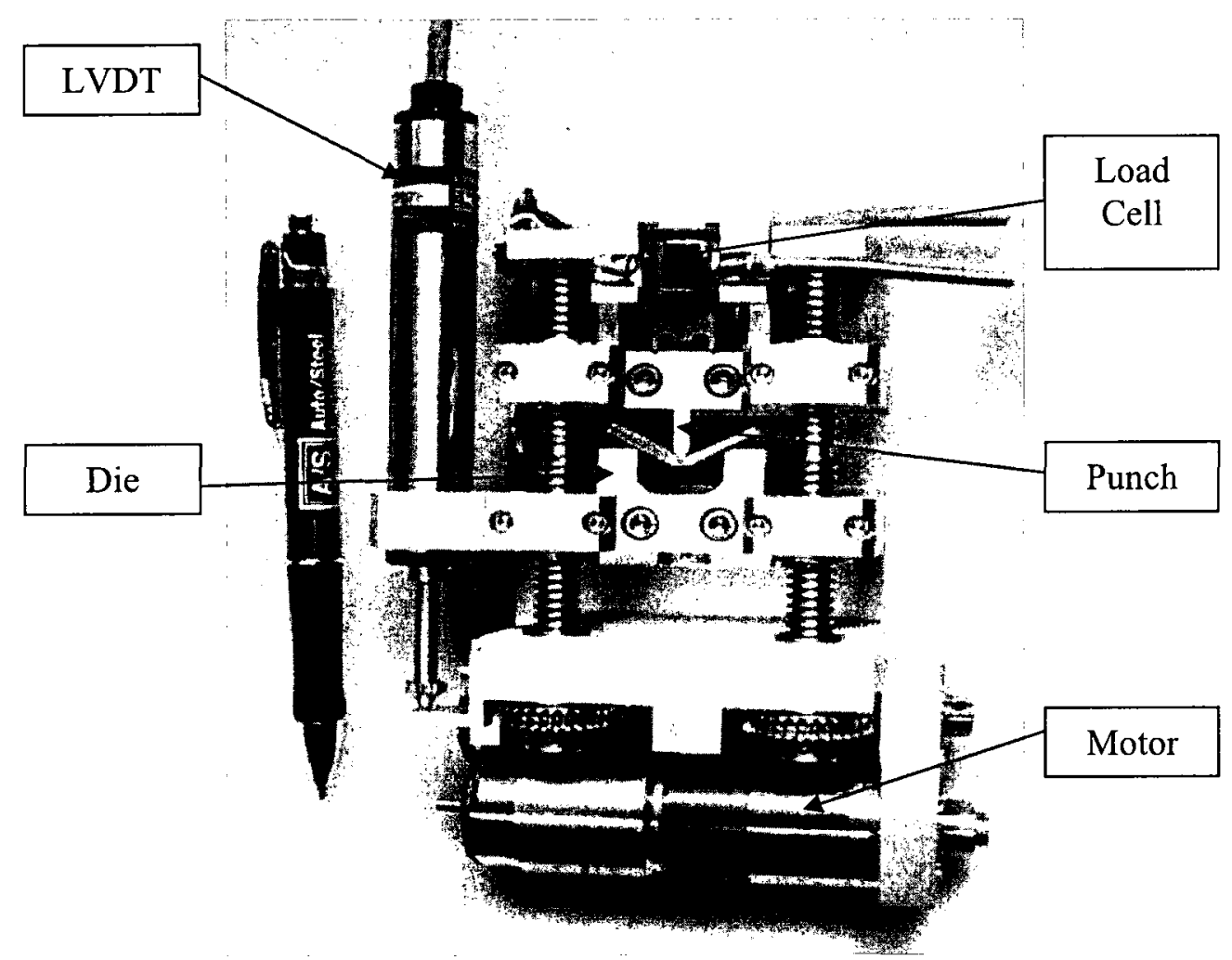

Figure 2-1: Loading Stage [11]. 


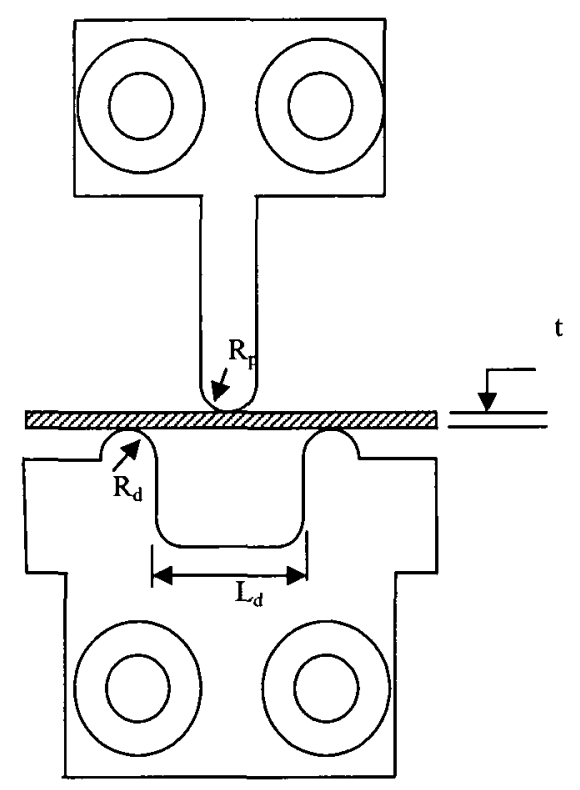

Figure 2-2: Schematic of microbending tooling.

\subsection{Preparing Specimen}

The bending tests were performed with CuZn30 sheets with thicknesses of $1.625 \mathrm{~mm}, 0.5$ $\mathrm{mm}$ and $0.25 \mathrm{~mm}$, which were selected to be identical to past research specimens so results could be compared. Also, CuZn30 is of interest to the electronics industry for microforming applications. The dimensions of the specimens were designed according to the theory of similarity also. To ensure a plane strain deformation, the width of the specimen was ten times the thickness (i.e., 10t). The specimens were heat treated to obtain different grain structures for the material with consistent numbers of grains through the thickness. All the specimens were heat treated under a pure argon gas environment for one hour. The ASTM E112 standard procedure [13] was used to 
determine grain size on the side of the specimens. Specimens with coarse, medium and fine grain sizes, corresponding to approximately $2-4,10-15$, and 25-30 grains through the thickness respectively were obtained. The specific grain size information is provided in Table 2-1.

\begin{tabular}{|c|c|c|c|c|c|c|}
\hline $\begin{array}{c}\text { Thickness } \\
(\mathrm{mm})\end{array}$ & $\begin{array}{c}\text { Measured } \\
\text { grain size } \\
(\mu \mathrm{m})\end{array}$ & $\begin{array}{c}\text { Standard } \\
\text { Deviation }\end{array}$ & $\begin{array}{c}\text { Measured } \\
\text { grains } \\
\text { through } \\
\text { thickness }\end{array}$ & $\begin{array}{c}\text { Grain } \\
\text { Structure }\end{array}$ & $\begin{array}{c}\text { Heat } \\
\text { treatment } \\
\text { Temperature } \\
\text { (degree } \mathrm{C})\end{array}$ & $\begin{array}{c}\text { Duration } \\
\text { (minutes) }\end{array}$ \\
\hline \multirow{3}{*}{1.625} & 64.8 & 7.37 & 25.2 & Fine & 565 \\
\cline { 2 - 6 } & 151.9 & 14.22 & 10.7 & Medium & 650 \\
\cline { 2 - 6 } & 706.5 & 74.28 & 2.3 & Coarse & 850 \\
\hline \multirow{3}{*}{0.500} & 17.2 & 1.47 & 29 & Fine & 400 \\
\cline { 2 - 6 } & 35.5 & 2.96 & 14.1 & Medium & 500 \\
\cline { 2 - 6 } & 142.9 & 12.33 & 3.5 & Coarse & 665 \\
\cline { 2 - 6 } 0.250 & 10.0 & 0.84 & 25.1 & Fine & 400 \\
\cline { 2 - 6 } & 22.9 & 1.65 & 10.9 & Medium & 500 \\
\cline { 2 - 6 } & 67.6 & 6.49 & 3.7 & Coarse & 665 \\
\hline
\end{tabular}

Table 2-1: Specimen thickness, grain size, and heat treatment information.

\subsection{Digital Image Correlation System}

Digital Imaging Correlation (DIC) is a non-contact, optical method to measure full-field, $2 \mathrm{D}$ or $3 \mathrm{D}$ measurements of displacement and strain by tracking the gray value pattern in consecutive images during deformation [14]. In our experiments, Vic-2D software from Correlated Solutions Inc. was used for these analyses. The DIC method provides a straightforward means to obtain the strain distribution from experiments. The main steps to conduct the tests are: 
- Preparing the specimen

- Setting up the camera

- Running the test

- Image analysis

- Post-processing the data

\subsubsection{Preparing the specimen for DIC Analysis}

Preparing the region of interest on the thin metal sheet specimen with a speckle pattern is very important, since the DIC technique relies on a contrasting pattern on the surface of the test specimen. To achieve effective correlation, the pattern must be non-repetitive and isotropic (i.e., the pattern should be random and not exhibit a bias to one orientation) with a high contrast between dark blacks and bright whites [15]. For microbending, the area of interest is the side of the metal sheet, which is approximately $4 \mathrm{t}$ in length (centered on the location directly under the punch radius) and through the entire thickness. The most common techniques for applying the black speckle pattern are with ordinary spray paint or carbon toner. However for very small specimens $(<1.5 \mathrm{~mm})$, a much finer pattern is required. Thus, a Professional Iwata CM-B Custom Micron Series Airbrush was used. The paint used was Medea Com-Art Airbrush Paints (due to the low viscosity) with black and white colors. A sample pattern on a $0.5 \mathrm{~mm}$ specimen is shown in Fig. 2-3. 


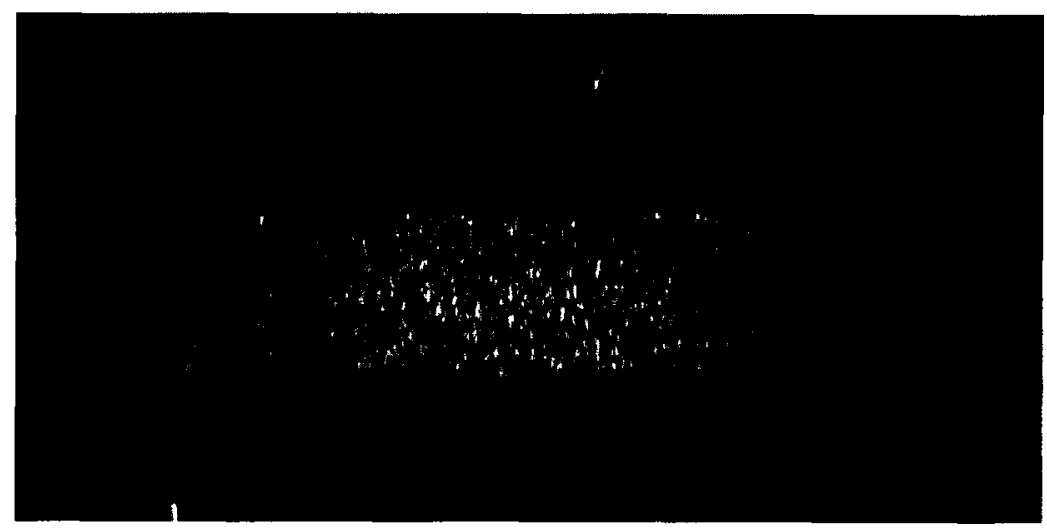

Figure 2-3: Pattern applied by airbrush on the surface of a specimen.

\subsubsection{Setting up the camera}

In microbending, a single camera can be used to measure the deformation since the deformation is predominantly in-plane. The lens for the $0.25 \mathrm{~mm}$ and $0.5 \mathrm{~mm}$ specimens had a $45 \mathrm{x}$ zoom magnification, and a Barlow 2.0x lens was added to increase the magnification further. A Tamron CCTV $35 \mathrm{~mm}$ lens was used for $1.625 \mathrm{~mm}$ thickness

specimens. A Point Grey GRAS-20S4M/C digital camera was used for all specimen sizes, with an ICX274 1/1.8" Image Sensor and pixel array size of $1624(\mathrm{H}) \times 1224(\mathrm{~V})$. Camera and lens were mounted with mounting rings on a steel board to assure the specimen is planar and parallel to the camera sensor, see Fig. 2-4.

\subsubsection{Post-processing of the DIC data}


Figure 2-5 shows the full field, strain distribution data that is obtained from the DIC analysis, which is the Lagrangian strain $\left(\varepsilon_{\text {lag }}\right)$. This data was converted to engineering strain $\left(\varepsilon_{\text {eng }}\right)$ for plastically-deforming material subjected to small deformation (which is an approximation here) using:

$$
\begin{aligned}
& \varepsilon_{\text {eng }}=\frac{\mathrm{L}-\mathrm{L}_{0}}{\mathrm{~L}_{0}} \\
& \varepsilon_{\text {lag }}=\frac{1}{2}\left(\frac{\mathrm{L}^{2}}{\mathrm{~L}_{0}^{2}}-1\right) \\
& \varepsilon_{\mathrm{eng}}=\sqrt{2 * \varepsilon_{\text {lag }}+1}-1
\end{aligned}
$$

All further figures in this thesis are of engineering strain values, since engineering strain line is linear through the thickness of specimen. The area of particular interest is the strain distribution directly under the punch, see line in Fig. 2-5. Strain distribution data was obtained from the DIC analysis when the specimen is in the fully loaded state. Thus, the data is prior to springback.

Note that while the entire thickness was selected as the area of interest for the DIC analysis, data is lost at the outer surfaces, particularly on the compression side of the bending process. This is due to the software not being able to correlate the speckle pattern in this region. On the compression side, this is likely due to excessive compression and damaged speckle pattern at the punch contact location as observed in Fig. 2-5. On the tension side, this is likely due to the subset size not being entirely located on the 
specimen. It is common in DIC analyses to not obtaining data up to the edge of the specimen.

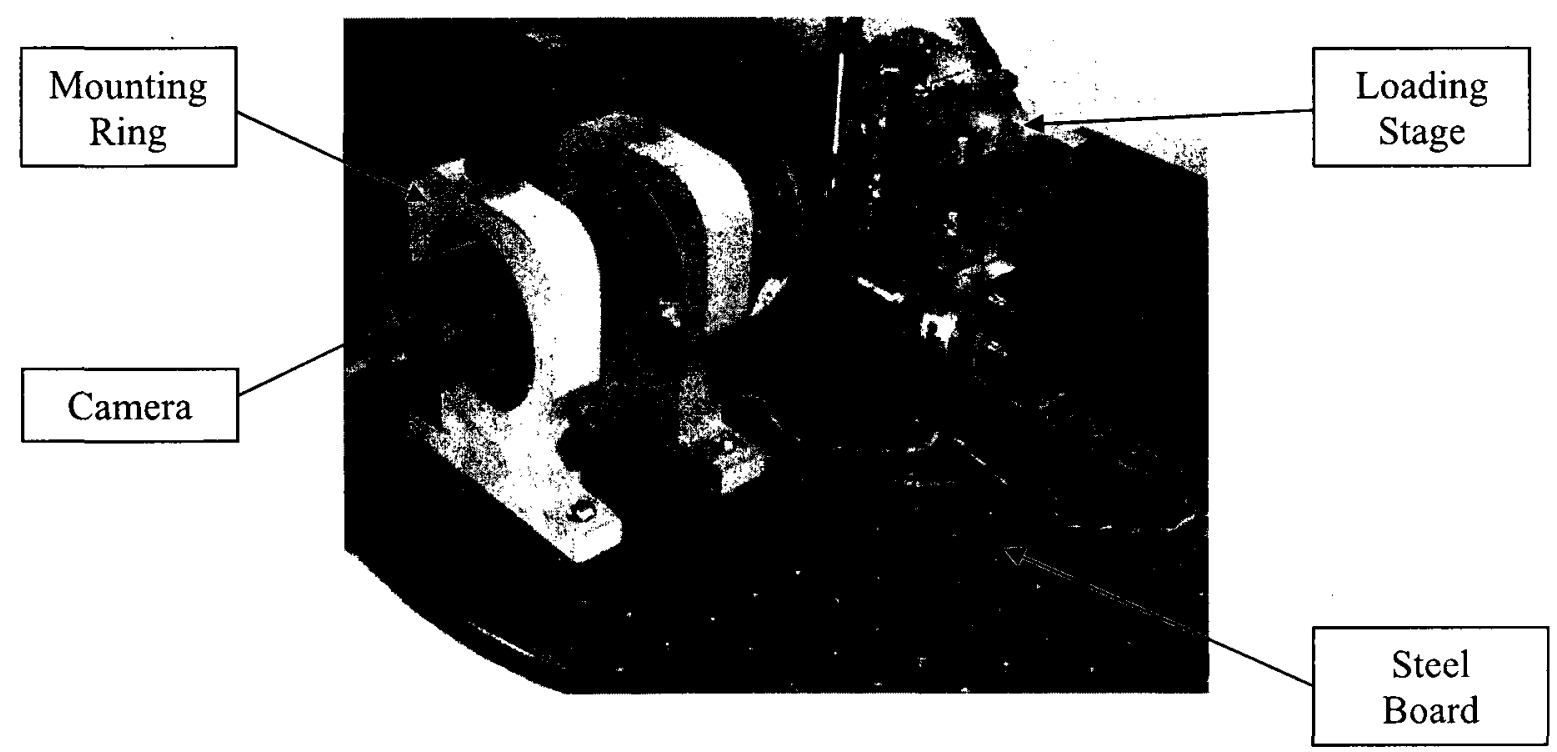

Figure 2-4: Set-up of camera, lens, and loading stage on a steel board.

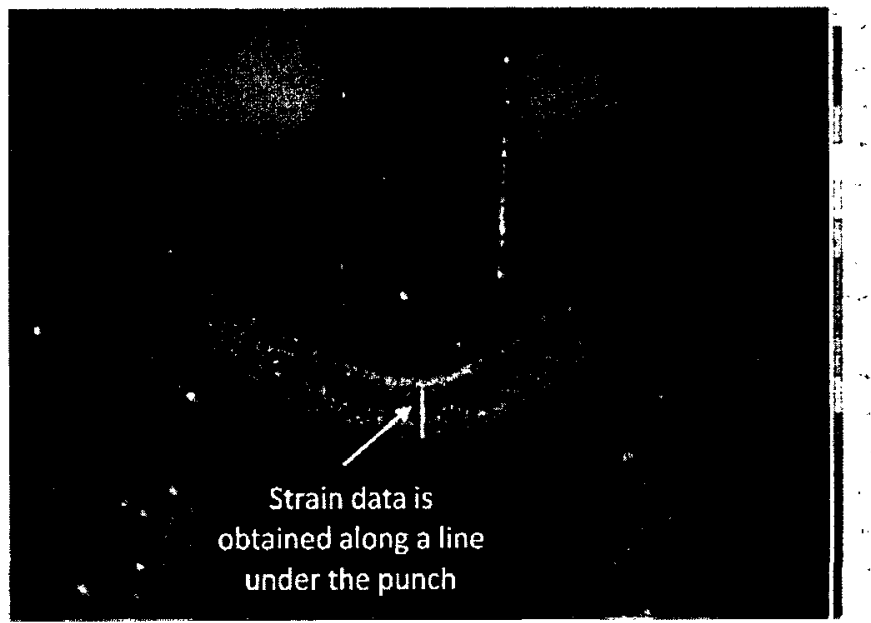

Figure 2-5: Full field, strain distribution data from DIC analysis. 


\subsection{Parametric study of DIC parameters}

To analyze the images with the DIC software, an effective subset and step size (measured in pixels of the camera) for the area of interest should be determined first, see Fig. 2-6. The subset size controls the size of the DIC analysis area and should be at least as big as the smallest speckle. The step size controls the density of the analyzed data (e.g., a step size of 5 will translate the center of the subset every 5 pixels during the analysis).

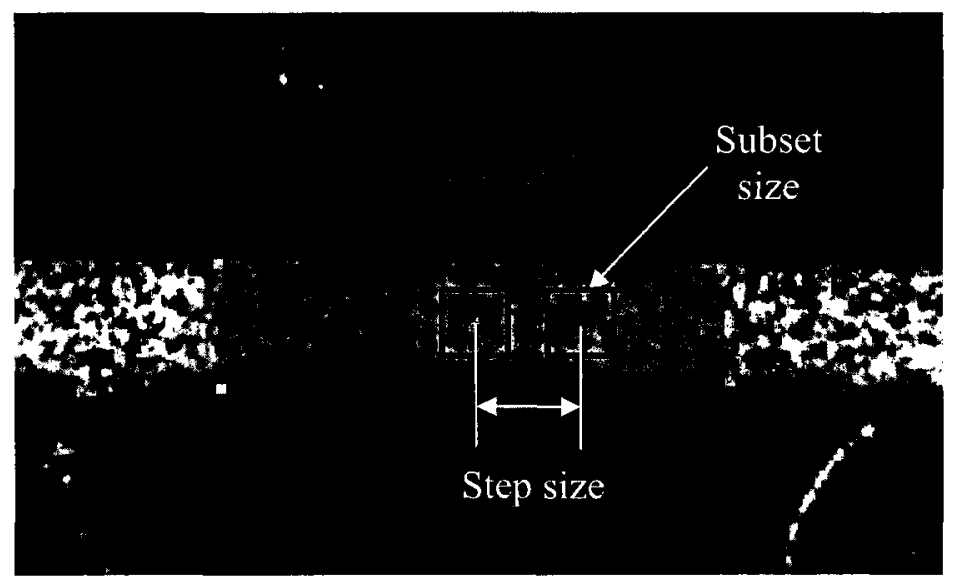

Figure 2-6: Schematic of subset and step sizes for DIC analysis.

After the DIC analysis, a dialog appears, which lists the number of points correlated, average iterations, confidence, and points per second. An average iteration value greater than 5 may indicate that the analysis has failed for all or part of the images. The confidence value is a one-standard-deviation confidence in the location of the match; thus lower numbers indicate better correlation. The approximate 0.02 value for confidence 
value was deemed acceptable for this application although lower values would have been preferred.

To investigate the effect of subset size and step size on the strain distribution results from the DIC analysis, a parameteric study of these parameters was conducted. The microbending experiment was performed with non-heat treated CuZn30 sheets of 1.625 $\mathrm{mm}$ and $0.5 \mathrm{~mm}$ thicknesses. The subset size was varied from 10 to 50 , while the step was varied from 1 to 11 . It was found that the average iterations and confidence varied with the change of subset size and step size, see Table 2-2. Also the strain distribution data was compared with various subset sizes from 20 to 40 with a constant step size at 3 of the $1.625 \mathrm{~mm}$ thick specimen, see Fig. 2-7 a). Similarly, the subset size was varied from 30 to 50 with a constant step size of 3 for the $0.5 \mathrm{~mm}$ thick specimen, see Fig. 2-7 b). These plots are the strain distribution directly under the punch, see line on Fig. 2-5, and are the average of three specimens. A total of 100 data points were obtained for this line of data (e.g., for the $0.25 \mathrm{~mm}$ specimen, strain data was obtained every $0.0025 \mathrm{~mm}$ ) which is significantly better resolution than the microhardness measurements in Parasiz et al. [11]. The limitation of this spatial resolution would be the pixel size of the image. The zero location on the $\mathrm{x}$-axis corresponds to the center of the sheets. The negative locations correspond to the compression side and the positive locations correspond to the tension side of the cross-section. It can be seen that the strain distributions are almost the same for both size specimen. Due to miniaturization, the $0.5 \mathrm{~mm}$ specimens show more variations.

Alternatively, when the strain distribution data was compared with varied step size and a constant subset value, (i.e., the step size varied from 1 to 7 with a constant subset size of 30 
and 40 for the $1.625 \mathrm{~mm}$ and $0.5 \mathrm{~mm}$ specimen respectively), the strain distribution deviates from each other. This result suggests that the strain distribution is more sensitive to variations in the step size, see Figs. 2-8 a) and b). From this parametric study, the step size was chosen to be 3 and the subset size was chosen to be 30 and 40 for the $1.625 \mathrm{~mm}$ and $0.5 \mathrm{~mm}$ thickness specimens respectively since these values provide relatively lower iteration and confidence numbers, see Table 2-2, which indicate a better correlation. Note that unlike finer mesh densities in finite element simulations the results do not necessarily have to converge when finer subset and step sizes.

a)

\begin{tabular}{|c|c|c|c|c|c|}
\hline Step & 1 & 3 & 5 & 7 & 11 \\
\hline Subset & & & & & \\
\hline 10 & 0.056 & 0.056 & 0.055 & 0.055 & 0.055 \\
& 2.6 & 3.0 & 3.3 & 3.5 & 3.9 \\
\hline 20 & 0.032 & 0.031 & 0.031 & 0.03 & 0.2 \\
& 1.9 & 2.0 & 2.3 & 2.5 & 3.0 \\
\hline 30 & 0.023 & 0.023 & 0.023 & 0.023 & 0.033 \\
& 2.0 & 1.8 & 2.1 & 2.4 & 2.8 \\
\hline 40 & 0.02 & 0.019 & 0.02 & 0.02 & 0.019 \\
& 2.1 & 2.0 & 2.2 & 2.5 & 3.0 \\
\hline 50 & 0.018 & 0.018 & 0.018 & 0.018 & 0.018 \\
& 2.2 & 2.2 & 2.5 & 2.8 & 3.4 \\
\hline
\end{tabular}

b)

\begin{tabular}{|c|c|c|c|c|c|}
\hline Subse Step & 1 & 3 & 5 & 7 & 11 \\
\hline 10 & & & & & \\
\hline 20 & 0.060 & 0.060 & 0.059 & 0.059 & 0.060 \\
& 2.6 & 3.0 & 3.3 & 3.5 & 3.7 \\
\hline 30 & 0.034 & 0.033 & 0.033 & 0.033 & 0.31 \\
& 2.1 & 2.4 & 2.5 & 2.7 & 3.3 \\
\hline 40 & 2.2 & 2.3 & 2.4 & 2.4 & 2.8 \\
\hline 50 & 0.024 & 0.024 & 0.024 & 0.024 & 0.024 \\
& 2.3 & 2.2 & 2.3 & 2.3 & 2.8 \\
\hline & 0.022 & 0.022 & 0.022 & 0.022 & 0.022 \\
& 2.7 & 2.5 & 2.6 & 2.6 & 3.3 \\
\hline
\end{tabular}

Table 2-2: Average confidence (top number) and iterations (bottom number) obtained with varying subset size and step size for a) $1.625 \mathrm{~mm}$ and b) $0.5 \mathrm{~mm}$ thickness specimens 

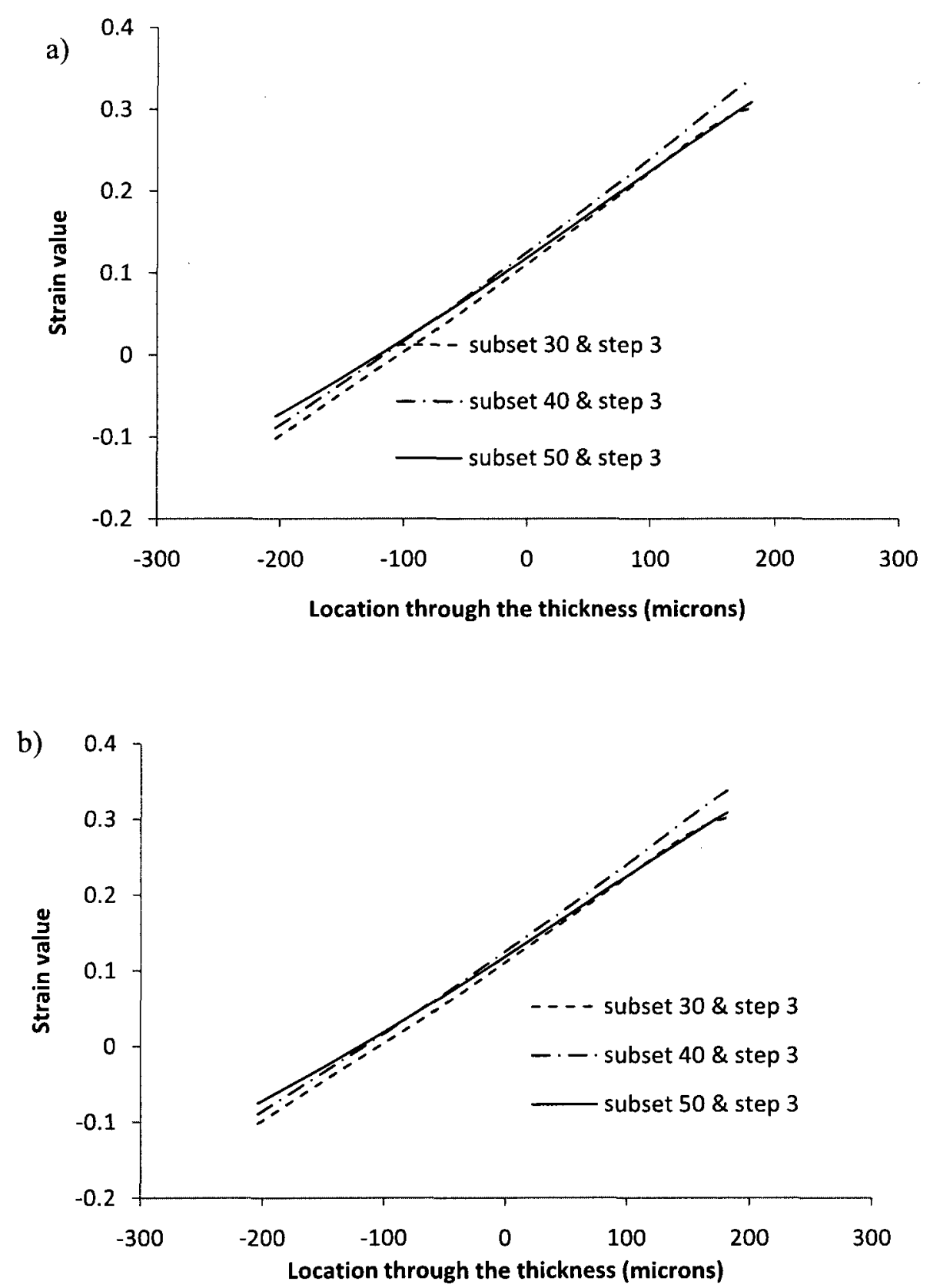

Figure 2-7: The strain distribution data with the varied subset size and specific step for a) $1.625 \mathrm{~mm} \mathrm{~b}) 0.5 \mathrm{~mm}$ thickness specimen. 

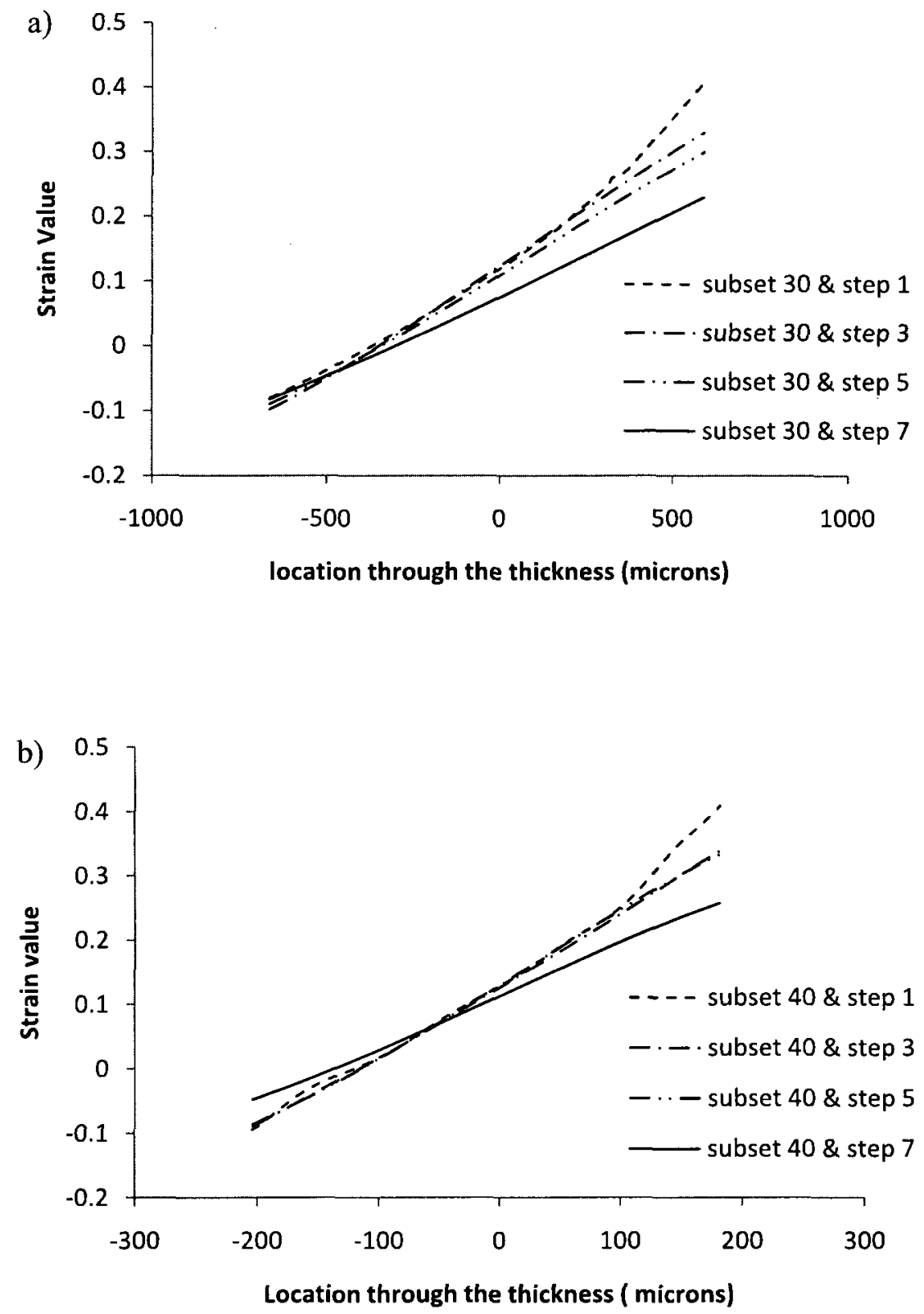

Figure 2-8: The strain distribution data with the varied step and specific subset size for a) $1.625 \mathrm{~mm} \mathrm{~b}) 0.5 \mathrm{~mm}$ thickness specimen.

\subsection{Loading Stage Modification}


Initially both of the plattens for the punch and die moved during microbending experiments. The neutral axis in the process (i.e., where the strain is zero) shifted significantly based on the DIC analysis. To evaluate if this was indeed correct, a microbending test with scribed lines through the thickness to physically measure the strain in the specimen was conducted. Several lines with $0.10 \mathrm{~mm}$ spacing were scribed through the thickness in the center area of a $0.5 \mathrm{~mm}$ specimen before the bending test, see Fig.2-9 a). After bending, by re-measuring the spacing between the lines, the neutral axis shifted by $0.074 \mathrm{~mm}$, see Fig. 2-9 b). Note that instead of increasing a linear distance for the $0.10 \mathrm{~mm}$ after bending, an arc length was used. The neutral axis shifting from the DIC system was $0.128 \mathrm{~mm}$ and the strain values at various locations did not match, see Table 2-3. The cause of this is the deformation area translating during the process.

To correct for this, the loading stage was adjusted by fixing the punch and thus providing for a stationary deformation area. A picture of the loading stage after this modification is provided in the Fig. 2-10. A microbending test with a $0.5 \mathrm{~mm}$ scribed lines specimen was conducted again. The neutral axis shift amount from the DIC, almost matched the strain results from scribed lines, $0.079 \mathrm{~mm}$ versus $0.071 \mathrm{~mm}$ respectively. Also, the strain value measured at various locations through the cross-section matched reasonable well, see Table 2-4. Errors in the scribed line measurements account for the differences in the two measurement methods. These measurements would correctly be characterized as estimates; however, sufficient confidence in the DIC method was obtained to proceed with the experiments. Note that the results from the DIC analysis were obtained under a loaded condition, while the results from scribed lines were obtained under an unloaded 
condition. However, the comparison is still meaningful since the elastic strain is small. Also note that while the images are blurry at the surfaces (see Fig 2-9), linear strain path are obtained as expected (see Fig 2-7 and 2-8) so the correlation with the DIC system remains reasonable.

a)

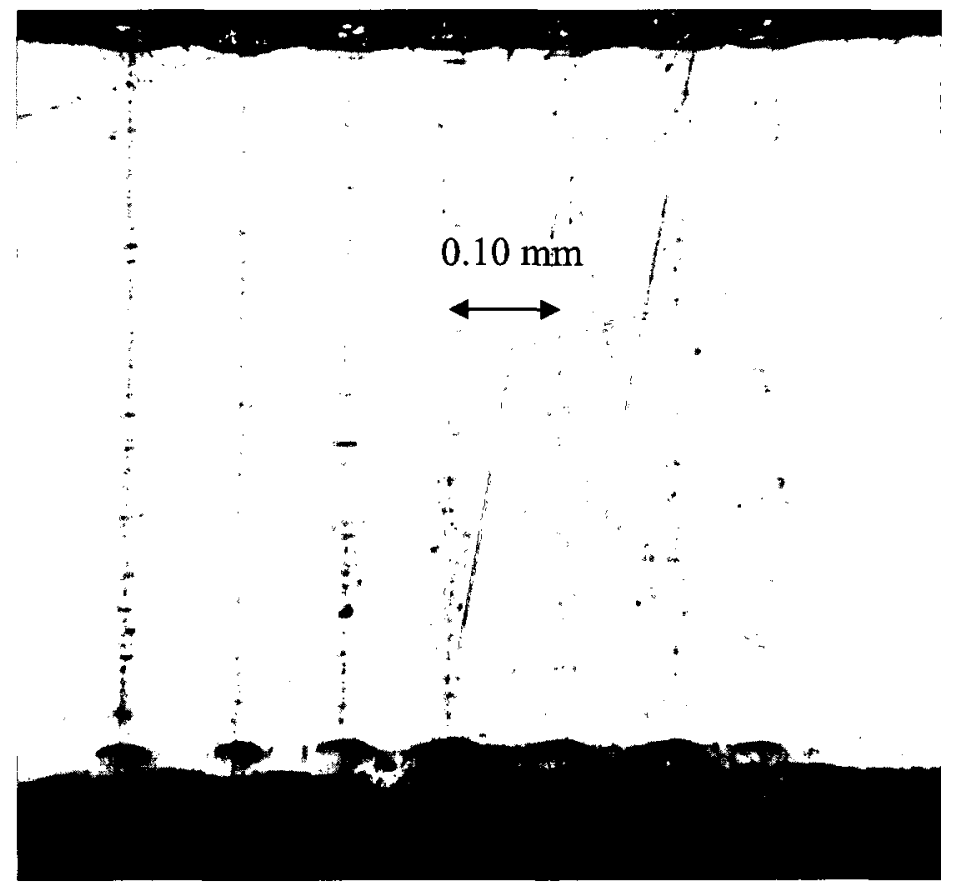


b)

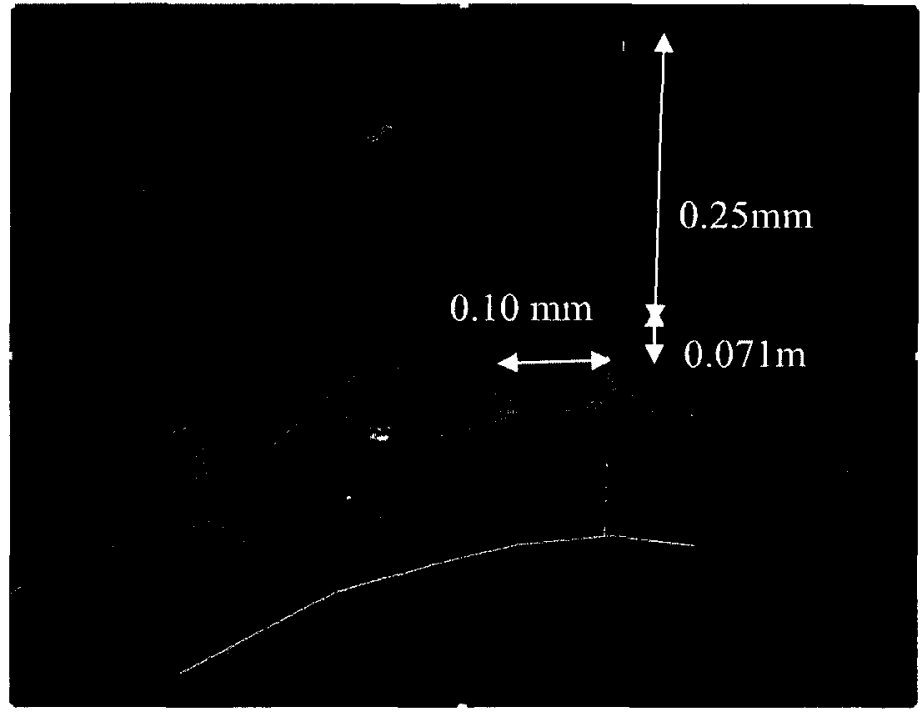

Figure 2-9: Specimen with scribed lines (a) before bending and (b) after bending.

\begin{tabular}{|c|c|c|c|}
\hline Location(mm) & $\varepsilon_{\text {DIC }}$ & $\varepsilon_{\text {lines }}$ & $\begin{array}{c}\text { Strain Difference } \\
\left|\varepsilon_{\text {DIC }}-\varepsilon_{\text {lines }}\right|\end{array}$ \\
\hline-0.15 & -0.007 & -0.092 & 0.085 \\
\hline-0.10 & 0.035 & -0.028 & 0.063 \\
\hline 0 & 0.124 & 0.113 & 0.011 \\
\hline 0.05 & 0.167 & 0.180 & 0.013 \\
\hline 0.10 & 0.211 & 0.238 & 0.027 \\
\hline 0.15 & 0.253 & 0.326 & 0.073 \\
\hline
\end{tabular}

Table 2-3: Strain values at various locations of the $0.5 \mathrm{~mm}$ specimen from DIC analysis results and scribed lines measurement before loading stage modification. 


\begin{tabular}{|c|c|c|c|}
\hline Location(mm) & $\varepsilon_{\text {DIC }}$ & $\varepsilon_{\text {lines }}$ & $\begin{array}{c}\text { Strain Difference } \\
\left|\varepsilon_{\text {DIC }}-\varepsilon_{\text {lines }}\right|\end{array}$ \\
\hline-0.15 & -0.093 & -0.089 & 0.004 \\
\hline-0.10 & 0.027 & -0.023 & 0.004 \\
\hline 0 & 0.103 & 0.106 & 0.003 \\
\hline 0.05 & 0.168 & 0.172 & 0.004 \\
\hline 0.10 & 0.232 & 0.237 & 0.005 \\
\hline 0.15 & 0.291 & 0.324 & 0.013 \\
\hline
\end{tabular}

Table 2-4: Strain values at various locations of the $0.5 \mathrm{~mm}$ specimen from DIC analysis results and scribed lines measurement after loading stage modification.

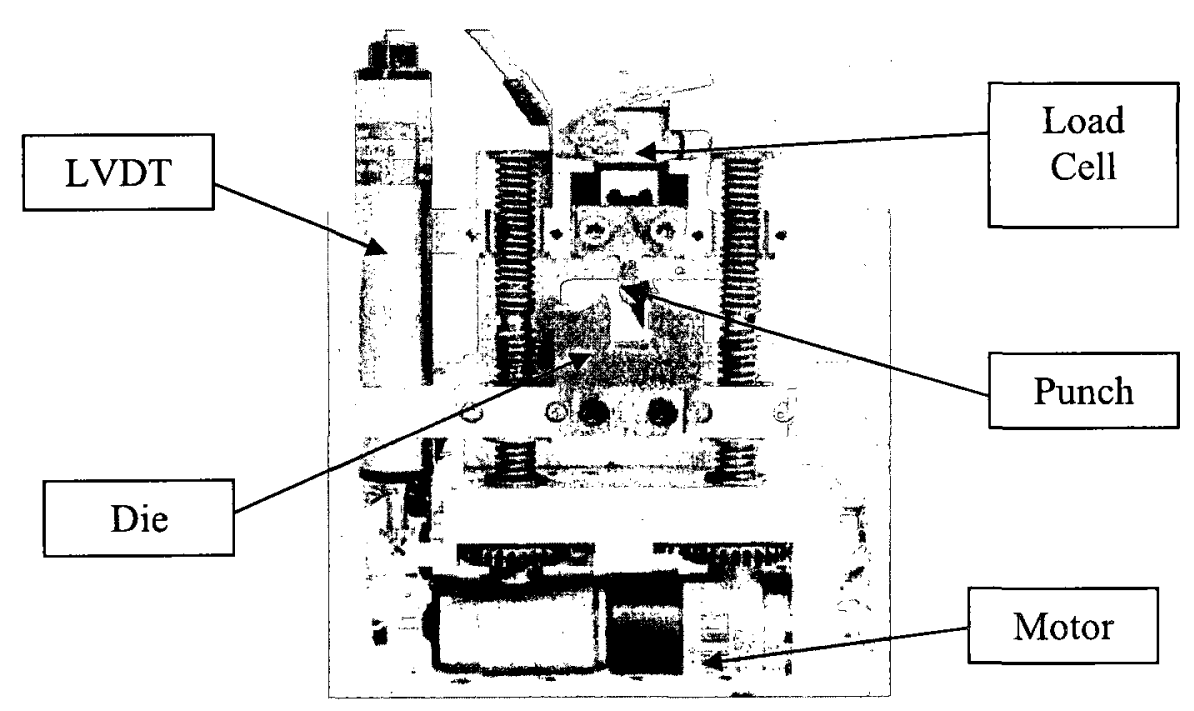

Figure 2-10: Loading stage after modification. 
Hill [8] provided a theoretical calculation of neutral axis location (i.e., fiber length change is zero during the bending operation) for bending of a wide sheet where there is negligible strain in the width direction. It is also assumed that the bending is achieved by moment couples applied at opposite ends of the specimen and that there are no other external forces. Initially during bending, the neutral axis is located in the center of the sheet. However, during the bending process, the neutral axis shifts to the compression side of the sheet, see Fig. 2-11). The radius of the neutral surface after bending is:

$$
\gamma=\sqrt{\mathrm{ab}}
$$

where $a$ and $b$ are the inner and outer radii of curvature respectively. Note that Eq.(2.4) is only an approximation provided the final radius of curvature is not less than four or five times the thickness of the sheet. Thus, the analytical model does not match our case where the final radius is 1.5 times the specimen thickness. The theoretical predicted shifting of the neutral axis based on this model is $0.0635 \mathrm{t}$ or $0.032 \mathrm{~mm}$. While this theoretical prediction does not match well the experimental neutral axis shifting amount $(0.07 \mathrm{~mm})$, it does indicate that the neutral axis shifts during bending and the shift amount can be theoretically predicted. Since the experimental punch radius $(1.5 \mathrm{t})$ is much tighter than that of the model $(4 t-5 t)$, the experimental neutral axis shifting is greater. 

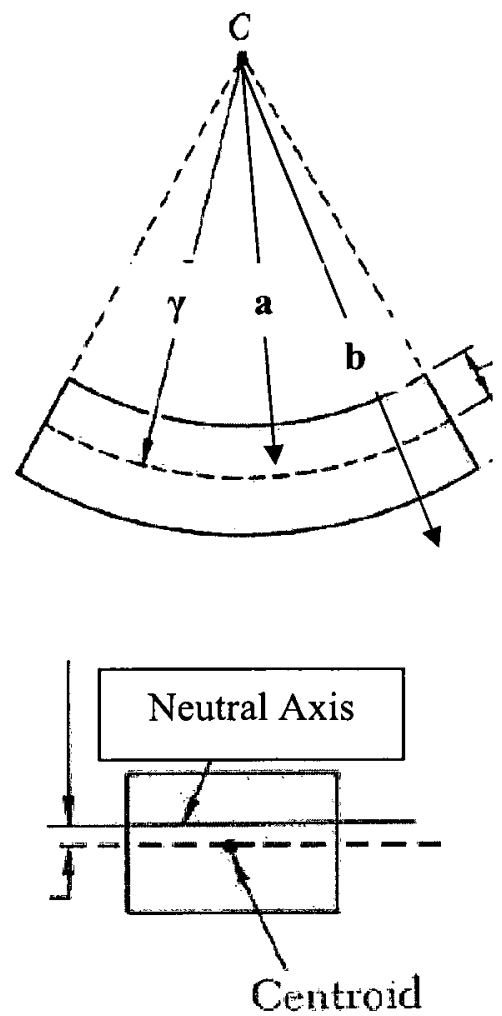

Figure 2-11: Schematic of the neutral axis location after bending. 


\section{CHAPTER 3}

\section{RESULTS OF MICROBENDING EXPERIMENTS}

Polycrystalline materials strain harden (i.e., their strength increases) due to deformation during bending processes. The more severe the plastic deformation (e.g., the sharper the bend radius), the higher the absolute strain value of the material after deformation. In bending operations, plastic strain gradients exist through the thickness of the workpiece. The deformation distribution can be characterized by a strain evaluation through the cross-section of the workpiece.

\section{$\underline{3.1 \text { Results }}$}

To investigate the effect of grain size and specimen size on deformation during microbending and to compare the results with those obtained by hardness measurements in Parasiz et al. [11], the strain distribution (i.e., the average of three specimens formed) through the thickness of the specimens is plotted in Fig. $3-1$ for the $1.625 \mathrm{~mm}, 0.5 \mathrm{~mm}$ and $0.25 \mathrm{~mm}$ specimens with varying grain sizes. An average strain distribution directly under the punch of three specimens is presented in each plot. In these figures, the zero location on the $\mathrm{x}$-axis corresponds to the center of the sheets. The negative locations correspond to the compression side and the positive locations correspond to the tension side of the cross-section. 


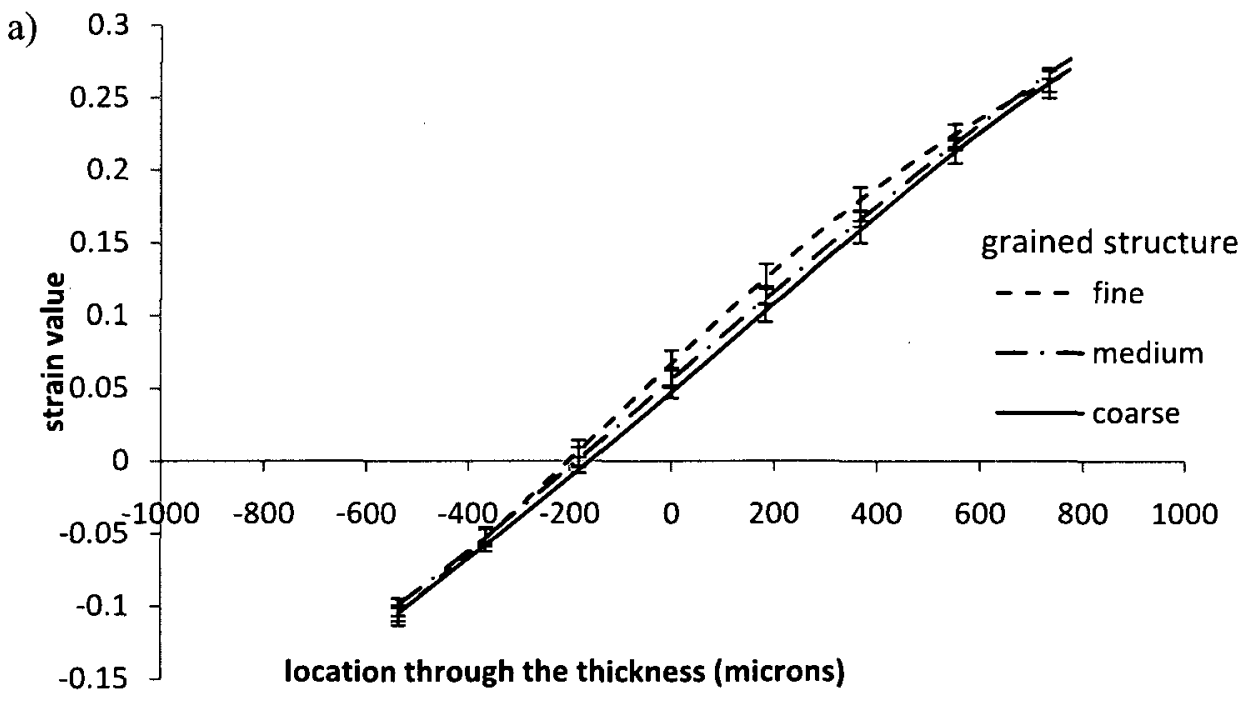

b)

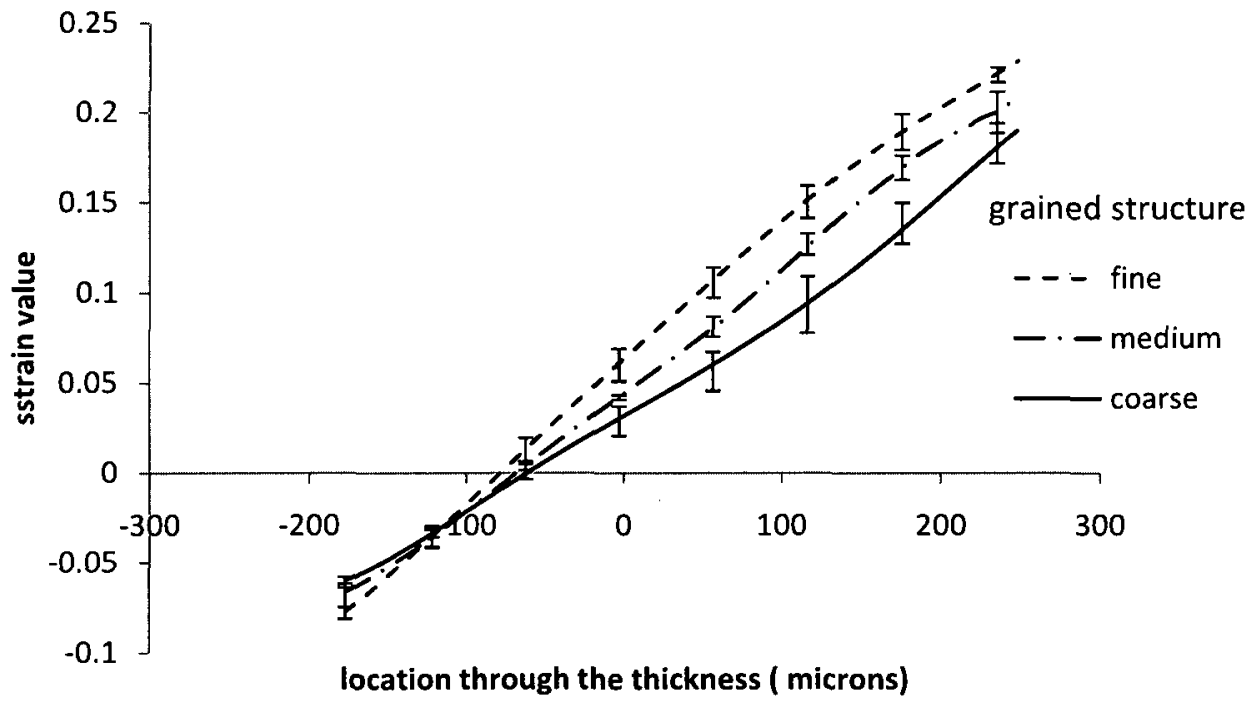


c)

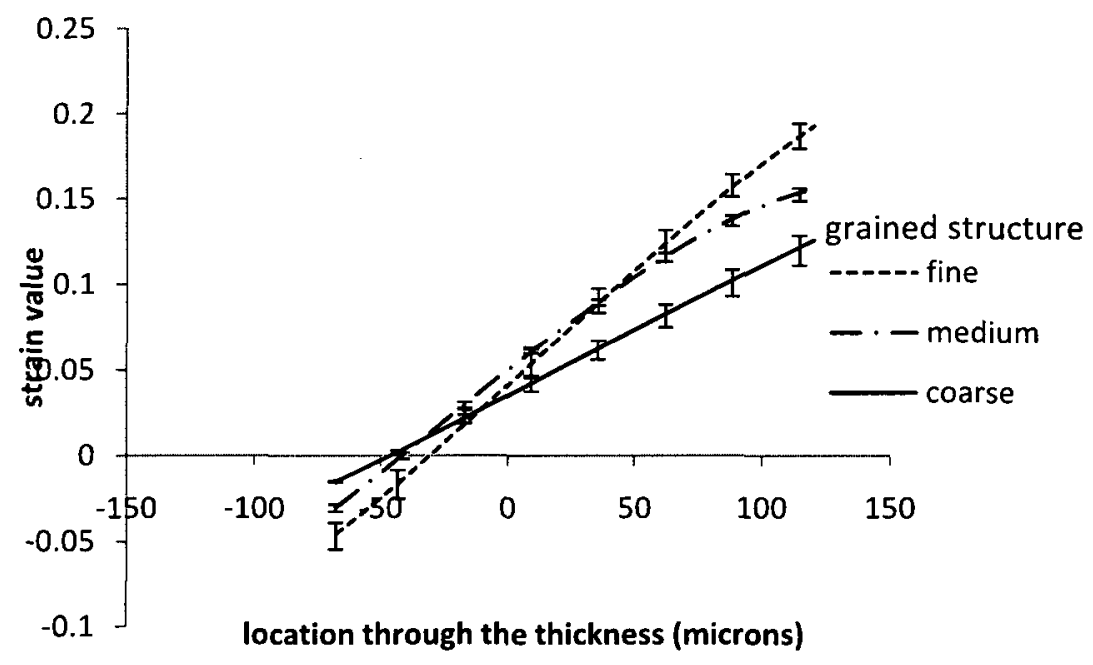

Figure 3-1: Strain distribution at the bend area directly under the punch through the thickness of a) $1.625 \mathrm{~mm}$, b) $0.5 \mathrm{~mm}$ and c) $0.25 \mathrm{~mm}$ sheets.

\begin{tabular}{|c|c|c|c|}
\hline \multicolumn{2}{|c|}{ Neutral Axis Shift $(\mathrm{mm}) /$ Average Ratio of Shift Amount to Specimen Thickness } \\
\hline structure & \multicolumn{2}{|c|}{ Fine } & \multicolumn{2}{c|}{ Medium } & \\
\hline $1.625 \mathrm{~mm}$ & $0.2085 / 12.8 \%$ & $0.1823 / 11.2 \%$ & $0.1561 / 9.6 \%$ \\
\hline $0.5 \mathrm{~mm}$ & $0.06225 / 12.5 \%$ & $0.0620 / 12.4 \%$ & $0.0476 / 9.5 \%$ \\
\hline $0.25 \mathrm{~mm}$ & $0.0302 / 12.1 \%$ & $0.0414 / 16.6 \%$ & $0.045 / 18 \%$ \\
& & & \\
\hline
\end{tabular}

Table 3-1: Amount of neutral axis shifting for $1.625 \mathrm{~mm}, 0.5 \mathrm{~mm}$ and $0.25 \mathrm{~mm}$ thickness specimens with varying grained structures. 
In Fig. 3-1 a), the average strain distribution through the thickness of $1.625 \mathrm{~mm}$ specimens is given for varying grain structures (i.e., fine, medium and coarse grained structures). All of the curves are similar with respect to the strain gradient that exists and the neutral axis location (i.e., the location where the strain is zero). Neutral axis locations are provided in Table 3-1 for all specimen sizes and grain structures. The neutral axis does not shift from the thickness center considerably with varying grain structures; however, a pattern exists where the shift increases with decreasing grain size. To better compare these results to those of Parasiz et al. [11], the absolute strain values were plotted, see Fig. 3-2 a). The absolute strain value is highest at the outer surfaces of the sheet and decreases linearly from the surface region to the inner region. The deformation distribution is relatively independent of grain structure for the largest size $1.625 \mathrm{~mm}$ thickness specimens.

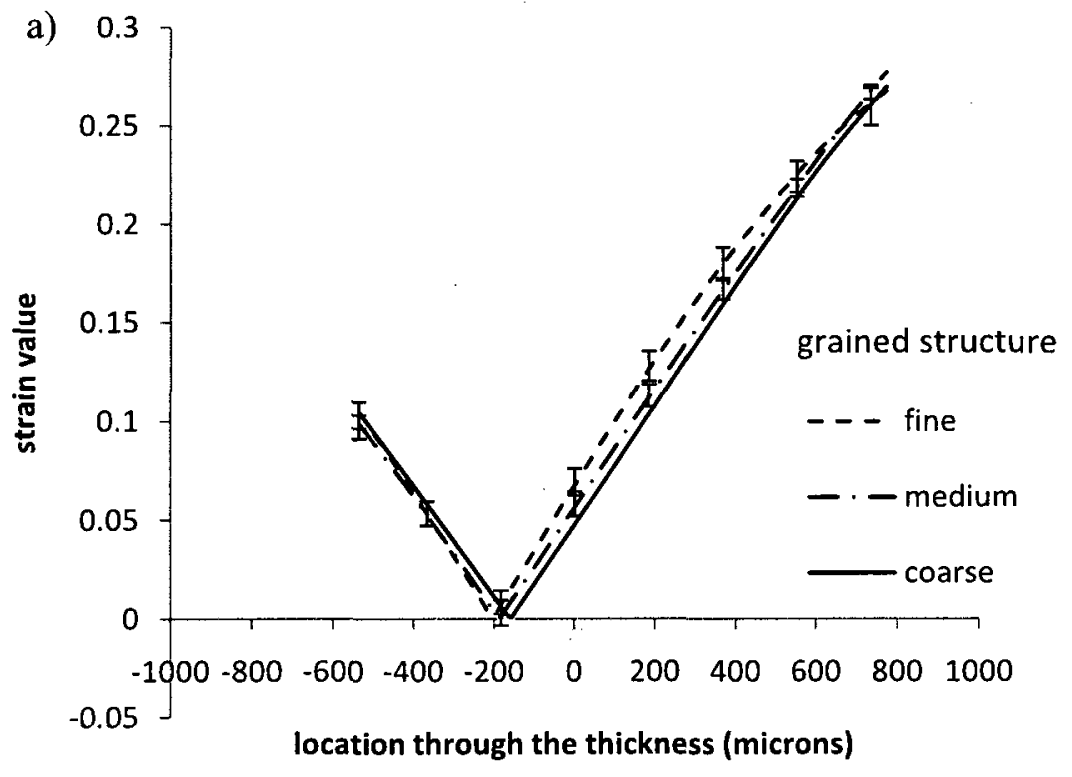



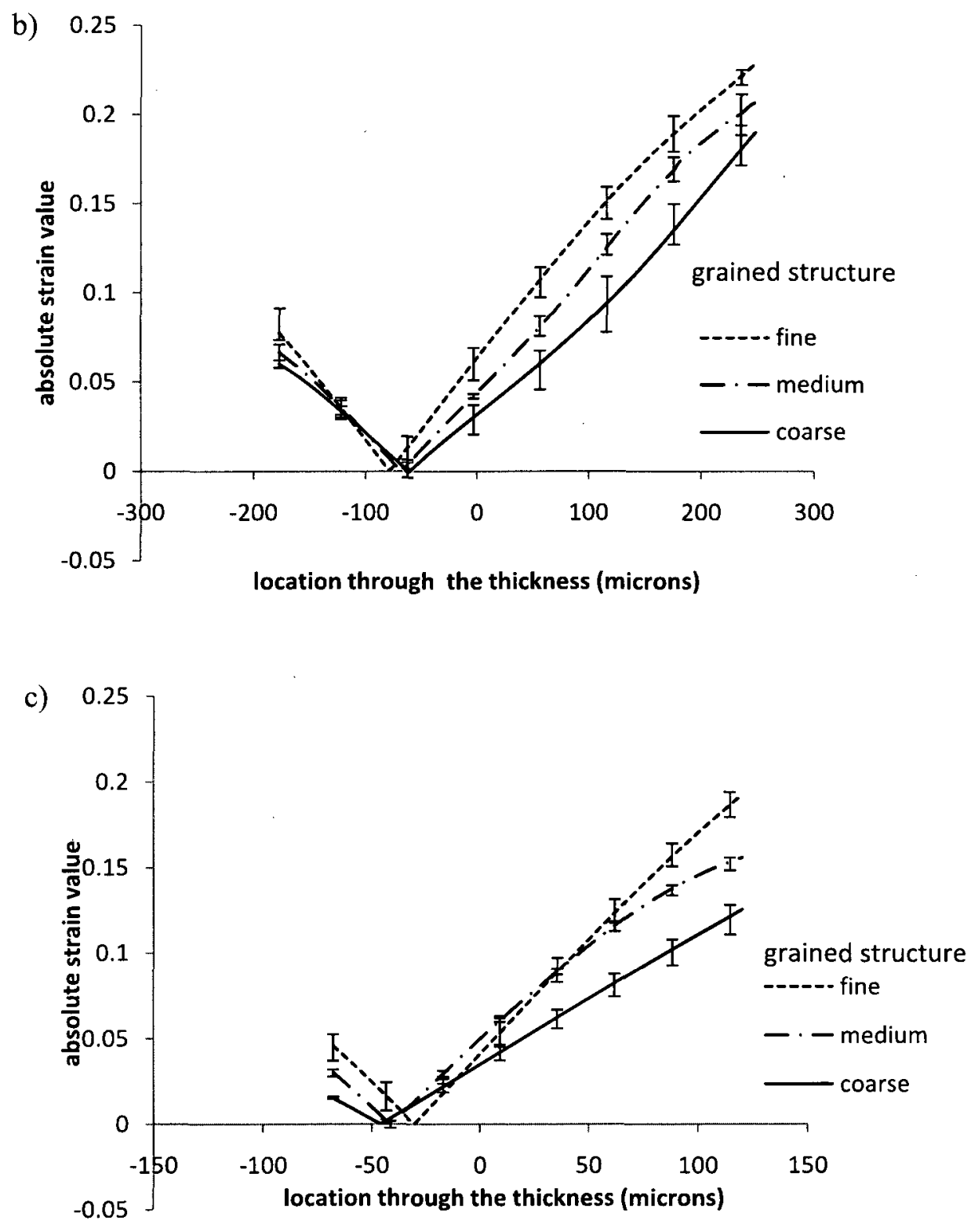

Figure 3-2: Absolute strain value distribution directly under the punch through the thickness for a) $1.625 \mathrm{~mm}$, b) $0.5 \mathrm{~mm}$ and c) $0.25 \mathrm{~mm}$ sheets with varying grain structures. 
In Fig. 3-1 b), the average strain distribution curves through the thickness of $0.5 \mathrm{~mm}$ specimens directly under the punch are given for all grain sizes. The strain gradient through the thickness is less steep with increasing grain size, producing higher strain values at the outer surfaces of the sheet for finer grained structures. This trend is similar to the hardness results presented in Parasiz et al. [11], see Fig. 1-4 as will be described further in the Discussion section. Note again that the neutral axis shifts towards the compression side after microbending. See Table 3-1 for locations of the neutral axis for $0.5 \mathrm{~mm}$ thickness specimens with varying grain structures. In Fig. 3-2 b), the absolute strain distribution curves for $0.5 \mathrm{~mm}$ thickness specimen are presented.

Finally, in Fig. 3-1 c), the average strain distribution curves through the thickness of the smallest size $0.25 \mathrm{~mm}$ thickness specimens are given. The effect of strain gradient through the thickness being less steep with increasing grain size is even more pronounced than for the $0.5 \mathrm{~mm}$ specimens. The shifting of the neutral axis shows an opposite trend than the $1.625 \mathrm{~mm}$ and $0.5 \mathrm{~mm}$ specimens with the neutral axis shift increasing with increasing grain size, see Table 3-1. In Fig 3-2 c), the absolute strain values for the 0.25 $\mathrm{mm}$ specimens are presented. This variation in the neutral axis location is another deformation size effect with miniaturization. Further comments on this will be provided in the Discussion Section.

To show the effect of specimen size on the strain gradient for each grain structure, the location through the thickness was normalized and the curves of all specimen sizes were plotted, see Fig. 3-3. The absolute strain value is shown in each plot. 
In Fig. 3-3 a), curves for the fine grained structure specimens are presented. The strain gradient is only slightly affected by the specimen size. However, for the medium and coarse grained structures shown in Figs. 3-3 b) and c) respectively, the strain gradient is less steep with miniaturization. Again, this trend is similar to the hardness results in Parasiz et al. [11], as will be described further in the Discussion section. The absolute strain difference between the $1.625 \mathrm{~mm}$ and $0.25 \mathrm{~mm}$ specimens at both the tension and compression outer surfaces for all grained structures are presented in Fig. 3-4. The strain value difference increases with increasing grain size due to the less steep strain gradients with miniaturization. To better understand the strain gradients with miniaturization, the strain gradient were assumed to be linear and the slope differences of the $1.625 \mathrm{~mm}, 0.5$ $\mathrm{mm}$ and $0.25 \mathrm{~mm}$ thickness specimens with varying grain structures are presented in Fig. 3-5. Note that the strain gradient slopes decrease as the grain size increases for each specimen size. However, the effect is most pronounced for the smallest $0.25 \mathrm{~mm}$ thickness specimens.

Though significant absolute strain difference with miniaturization can be obtained from DIC analysis results, almost no shape change can be observed from final shape of different size specimens with varying grain structures, see Figure 3-6. 

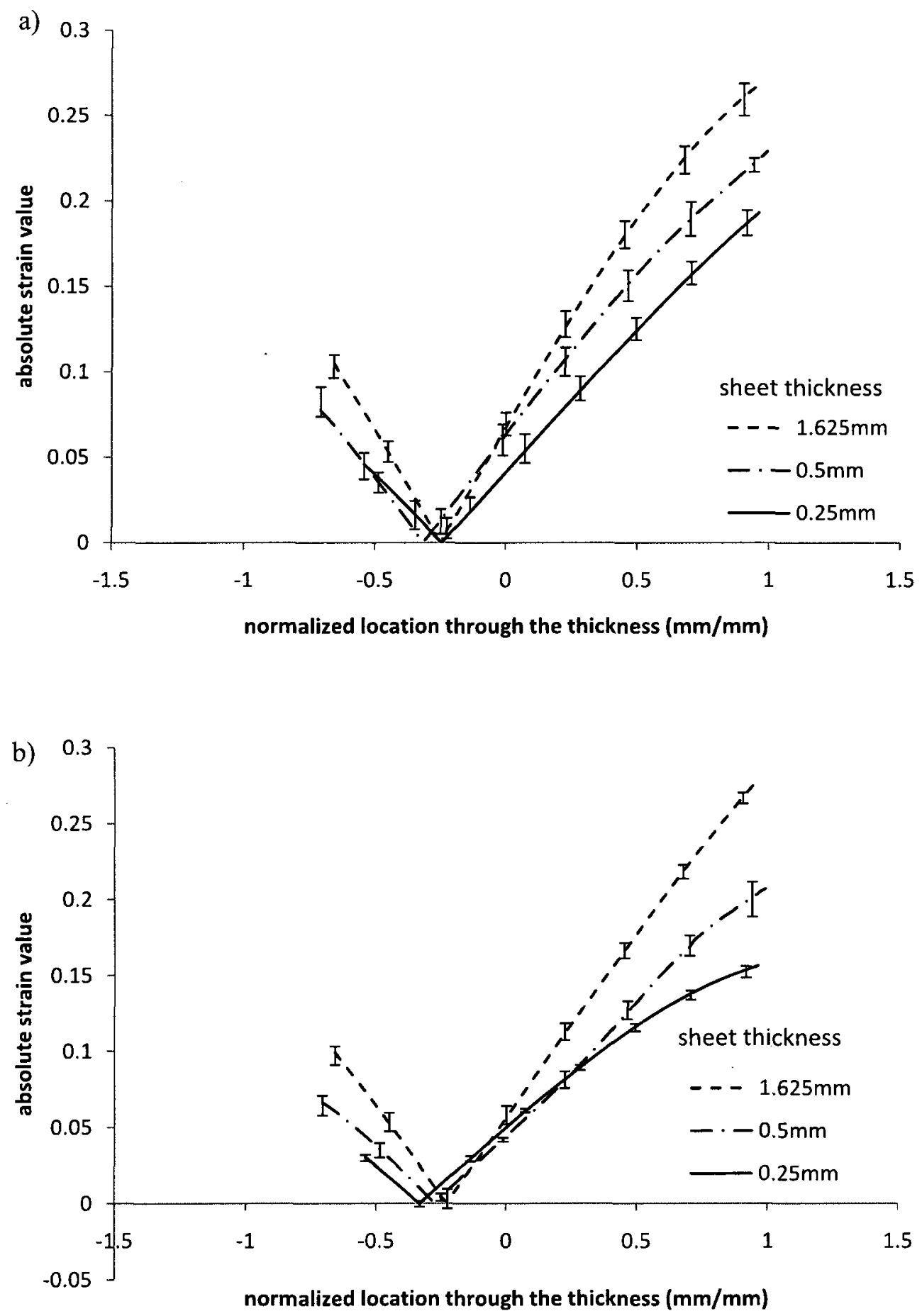
c)

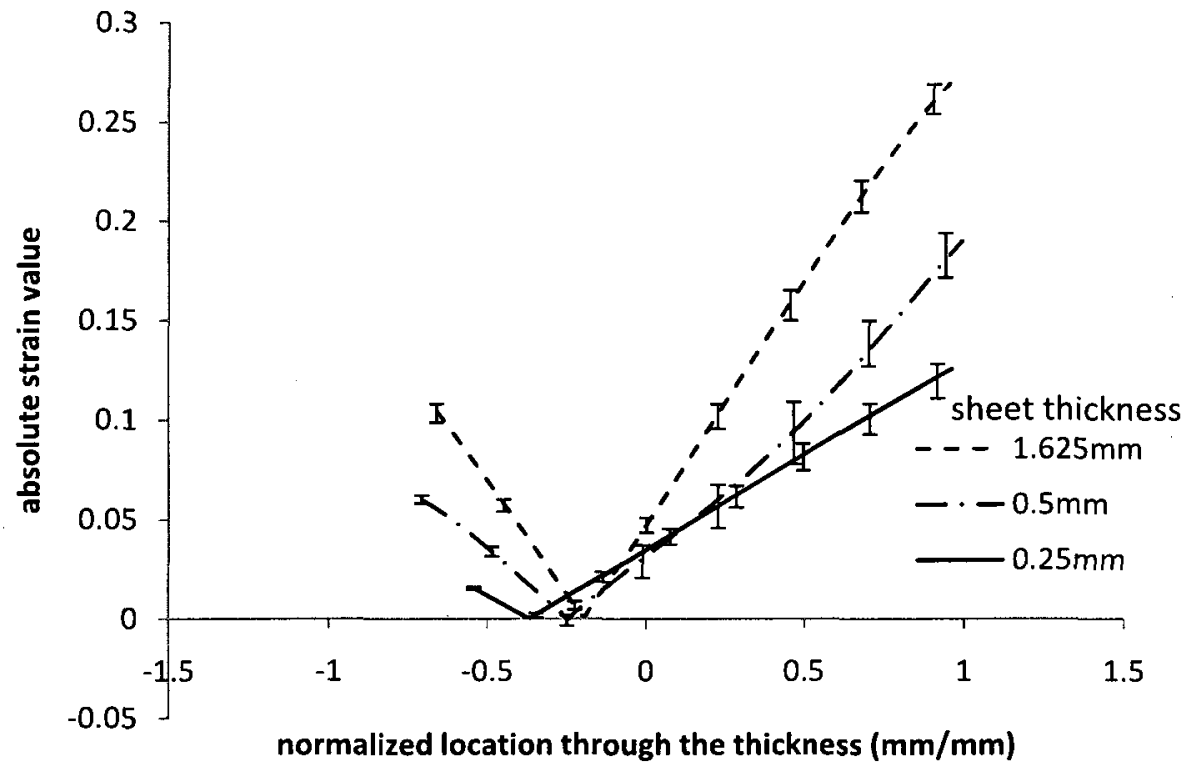

Figure 3-3: Absolute strain distribution through the thickness for the a) fine, b) medium and c) coarse grained structures.

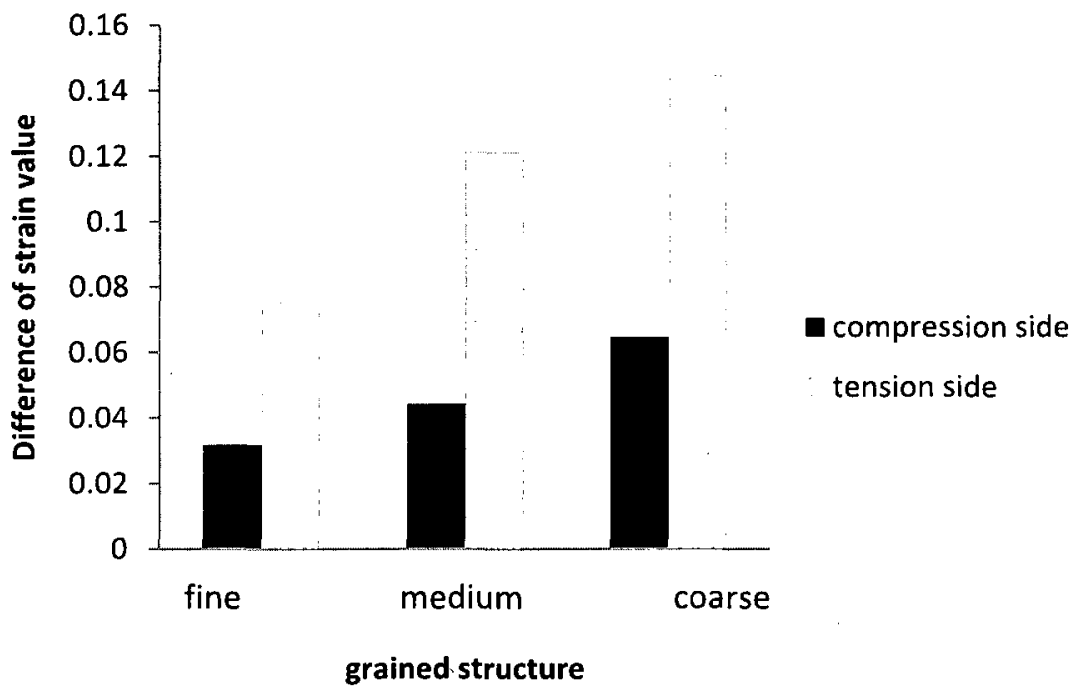

Figure 3-4: Absolute strain difference between the $1.625 \mathrm{~mm}$ and $0.25 \mathrm{~mm}$ specimens at outer fibers for varying grain structures. 


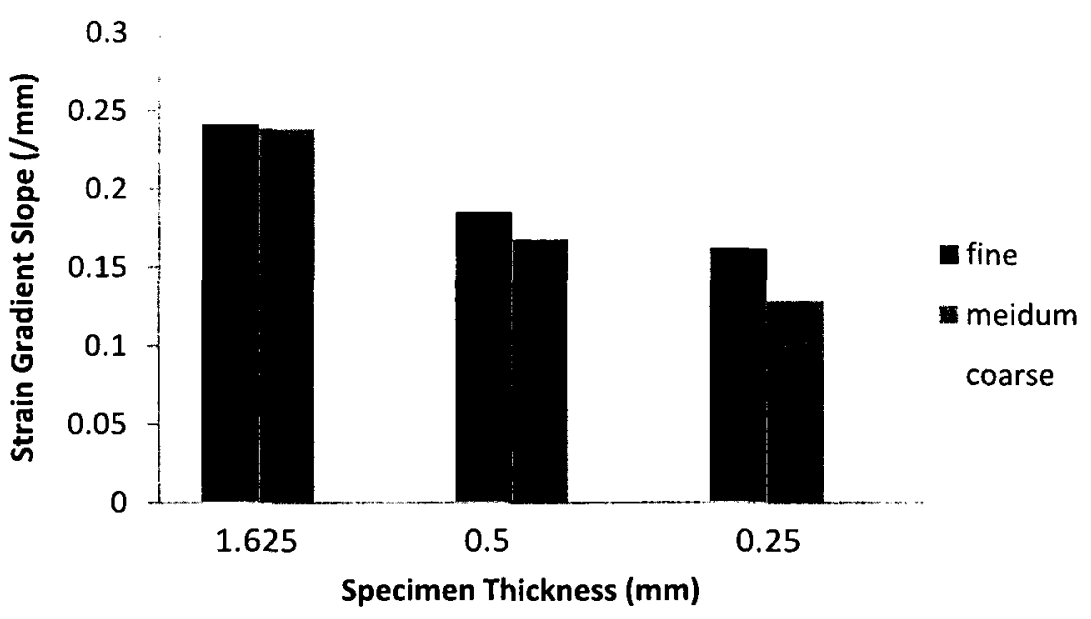

Figure 3-5: Strain gradient slope changes for $1.625 \mathrm{~mm}, 0.5 \mathrm{~mm}$ and $0.25 \mathrm{~mm}$ thickness specimen with varying grain structure.
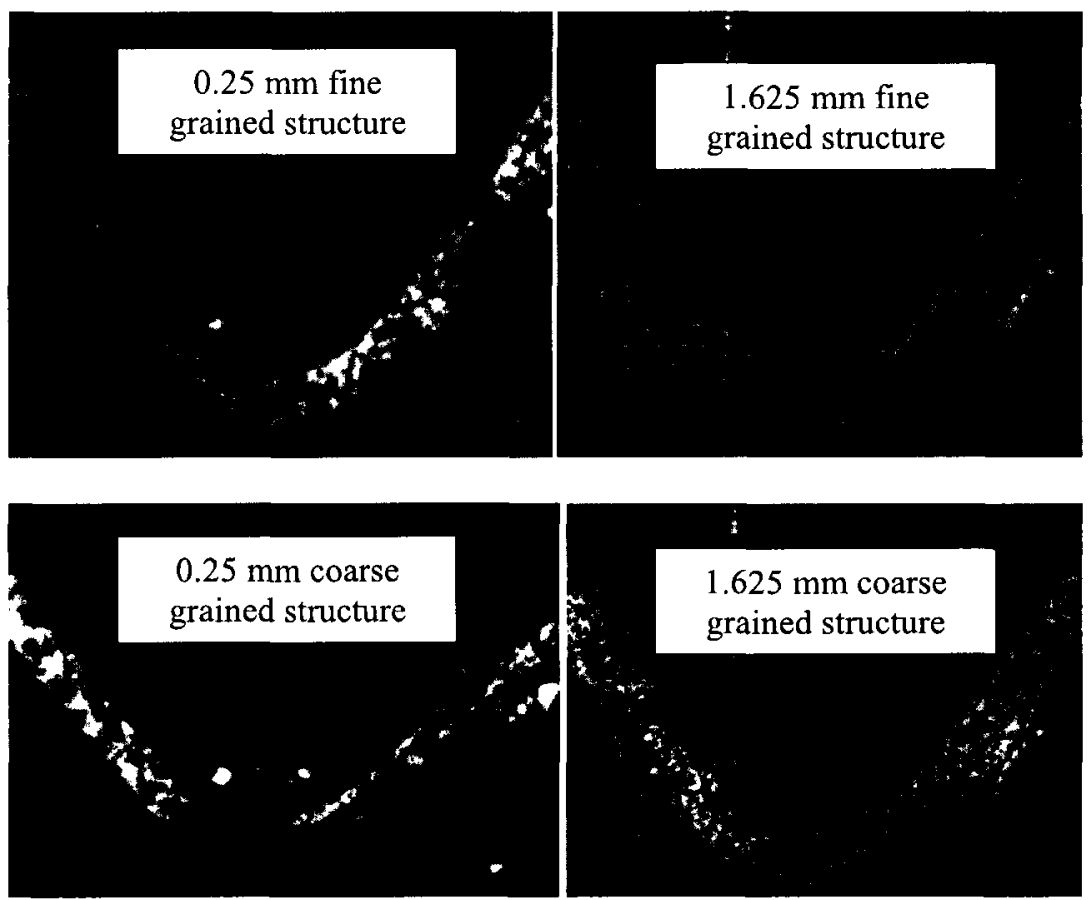

Figure 3-6: No shape change with miniaturization. 
- In addition to strain data, the bending force and punch displacement values, which were recorded by the load cell and LVDT respectively, are also of interest. The data was normalized so that the results from the three thicknesses could be compared. For the punch displacement, values were divided by the sheet thickness, since the punch displacement was 7 times the specimen thickness. Similarly, the bending force data was divided by the sheet thickness squared since bending force is a function of the sheet thickness squared [16]:

$$
P=\frac{c w t^{2} \sigma_{y}}{L_{d}}
$$

where $P$ is the maximum bending force, $c$ is a constant that varies from 0.3 to $1.3, w$ is the out-of-plane width of the sheet, $t$ is the sheet thickness, $\sigma_{y}$ is the yield strength of the material and $L_{d}$ is the die opening clearance. Recall that $w$ and $L$ are also functions of the thickness, i.e., $w=10 \mathrm{t}, L_{d}=7 \mathrm{t}$.

a)

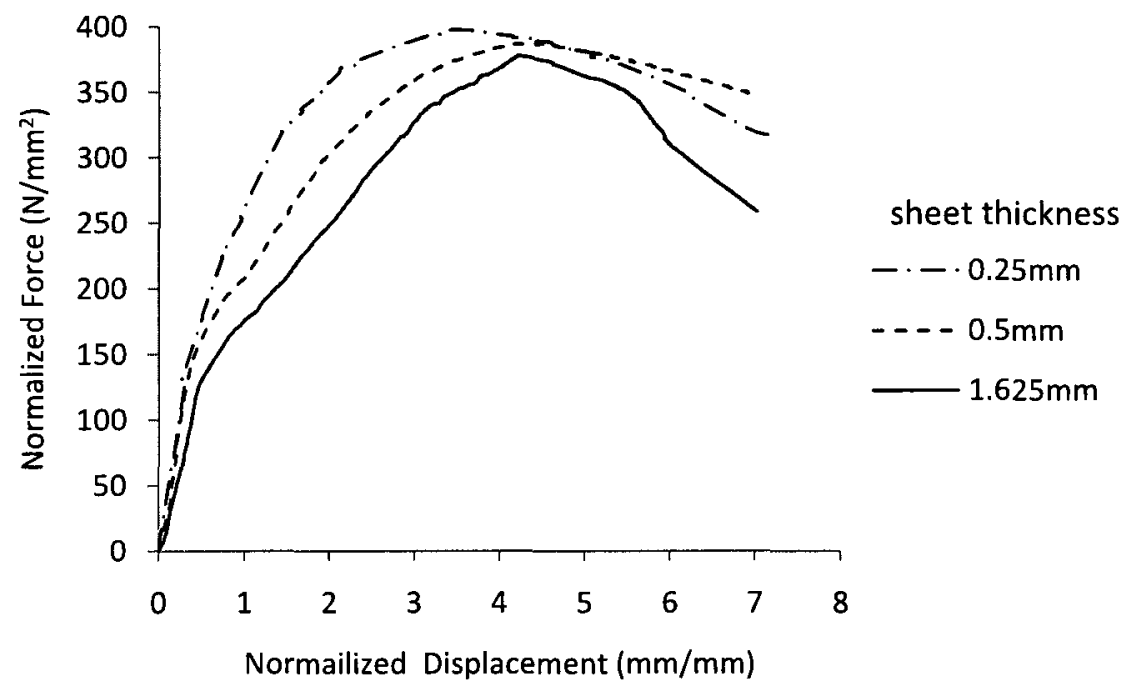


b)
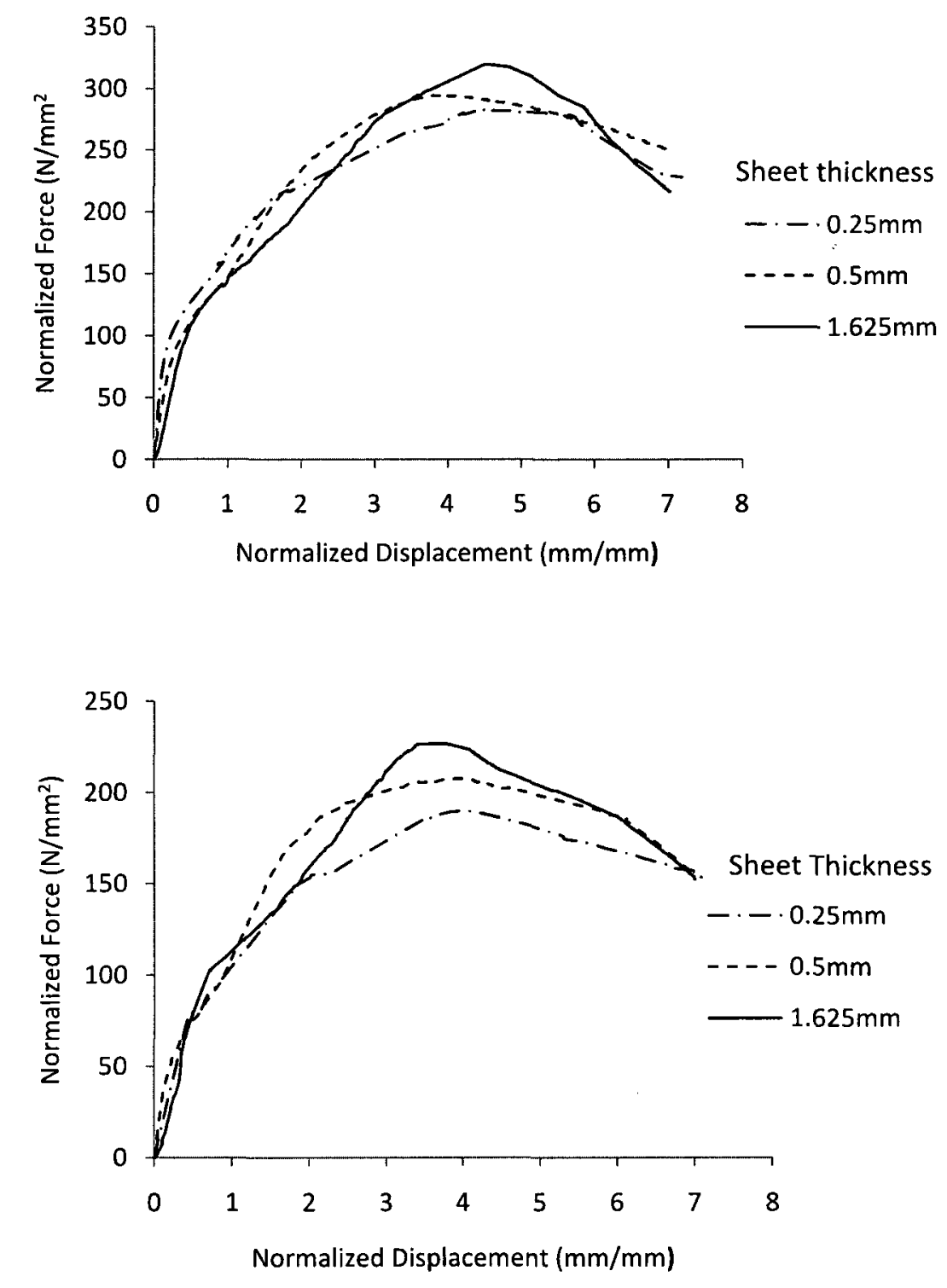

Figure 3-7: Normalized peak bending force versus specimen size curves for a) fine, $b$ ) medium and c) coarse grained structures.

Figure 3-7 shows the normalized bending force versus displacement curves for fine, medium and coarse grained structures. In Fig 3-7 a), for the fine grained specimens, the 
normalized peak force values increase slightly as specimen size decreases. However, for the medium and coarse grain structures in Figs. 3-7 b) and c) respectively, there is a slight decrease in the peak force as the sheet thickness is miniaturized from $1.625 \mathrm{~mm}$ to 0.25 mm. The normalized peak bending force versus specimen size curves for the three grained structures are shown in Fig. 3-8. This figure further shows that the normalized force decreases with miniaturization for the medium and coarse grained structures. The cause of this effect is unclear as the surface layer model [2] doesn't apply since all specimens have approximately the same number of grains through the thickness. Also, these results seemingly contradict the Hall-Petch effect as the $1.625 \mathrm{~mm}$ grain sizes are much larger than the $0.25 \mathrm{~m}$ grain sizes to achieve the same number of grain through the thickness (e.g., 2-4 in Fig. 3-7 c) for the coarse grained structures). Note that these results match those in Parasiz et al. [11]. This is believed to be a grain size effect which requires further study.

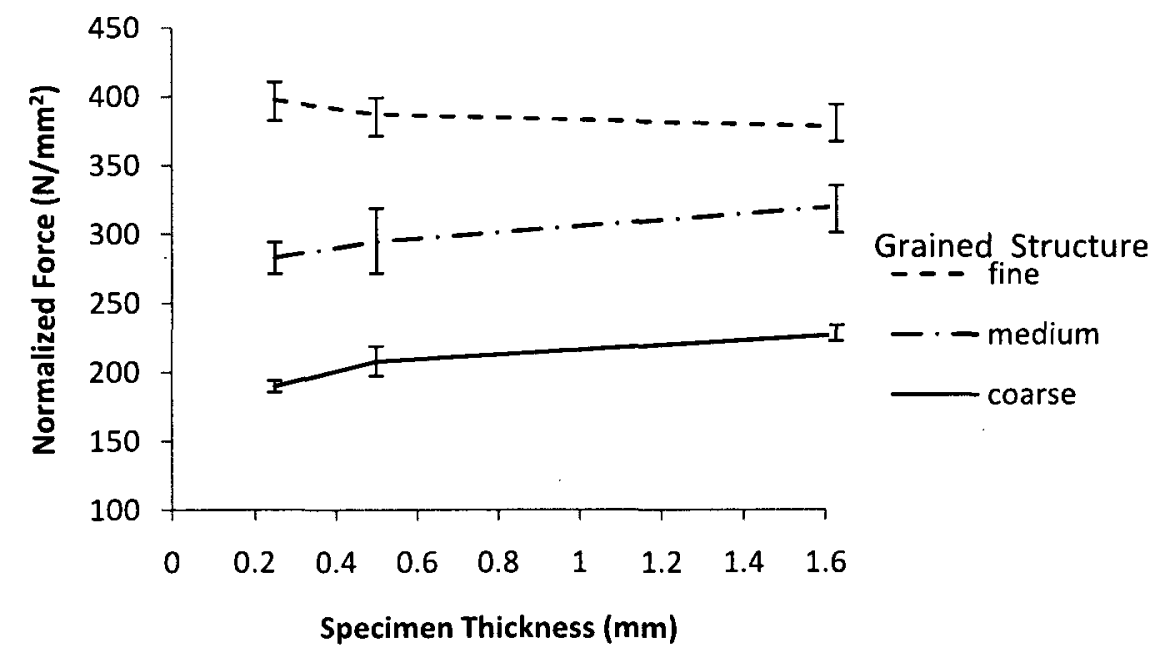

Figure 3-8: Normalized peak bending force versus specimen size curves for fine, medium and coarse grained structures. 


\subsection{Discussions}

The strain distribution results obtained from DIC analysis were compared to the previous results obtained by hardness measurement in Parasiz et al. [11] ( see Figure 3-9 and Figure 3-10), and the same trends were observed. The deformation distribution is less dependent on grain size for the largest size specimens (i.e., $1.625 \mathrm{~mm}$ ), while the grain structure has a significant effect on the strain distribution for the smaller size specimens (i.e., $0.5 \mathrm{~mm}$ and $0.25 \mathrm{~mm}$ thickness specimens). (Note that while hardness and strain are two distinct parameters, they both are related to the deformation of the material. With increased strain, the stress would increase due to work hardening and the hardness would increase as well. Of course, these relationships are not linear in nature so results are only compared with respect to the trends in the data.)

Similarly, for the fine grained structure specimens, miniaturization of the sheet thickness does not have a significant effect on the strain value distribution when compared to the medium and coarse grained structure ones. This trend is also similar to the hardness results of Parasiz et al. [11]. However, strain value differences for the three specimen sizes with fine grain structures was observed from DIC analysis result, see Fig 3-3 a), while it is not observed from hardness measurement result, see Fig 1-5 a). This is due to the relationship between stress (and hardness) and strain during plastic deformation. In the strain hardening region of the stress-strain curve, the stress value does not increase significant with increases in the strain value. The strain gradient exponent for the power hardening law of this material is approximately 0.3 [17]. 
Note that in this research, the number of grains through the thickness was used to represent the grain structure instead of the same grain size which was used in Parasiz et al. [11]. To investigate whether this change will affect the strain gradients observed, specific grain sizes and specimen sizes were chosen to compare the strain distributions i.e., 0.5 $\mathrm{mm}$ and $0.25 \mathrm{~mm}$ specimens with about $20 \mu \mathrm{m}$ grain size, $1.625 \mathrm{~mm}$ and $0.25 \mathrm{~mm}$ specimens with about $65 \mu \mathrm{m}$ grain size, and $1.625 \mathrm{~mm}$ and $0.5 \mathrm{~mm}$ with about $150 \mu \mathrm{m}$ grain size, see Table 2-1. The results are shown in Fig. 3-11. These results are similar to that of Fig. 3-3 with less steep strain gradients as the specimen size decreases with larger strain differences at the surface occurring for coarser grained specimens.

Assessing the deformation distribution with DIC was less time consuming than microhardness measurements and produced data for a more standard deformation parameter, i.e., strain. In addition, the neutral axis shifting was observed from the results of the DIC analysis, which can not be obtained in the hardness measurement results due to lack of continuous measurements. The trend of the neutral axis shifting can be explained by the grain size of the specimens and the penetration of deformation. For a given specimen size, a fine grained structure specimen has more grain boundary layers than the coarse structure one due to the smaller grain size. Thus, the neutral axis can shift across the grains more readily and thus a greater neutral shifting amount can occur. Furthermore, with miniaturization for a given grain size structure, deformation penetrates into the central region, in particular from the tensile surface, which shifts the neutral axis. However, further consideration and investigation of this effect are required. Finally, full field data is obtained from the DIC analysis. Figure 3-12 shows the strain distribution 
data for a $1.625 \mathrm{~mm}$ thickness specimen with a fine grain structure, see Fig. 3-12 a), and a $0.5 \mathrm{~mm}$ thickness specimen with a coarse grain structure, see Fig. 3-12 b). This figure shows that a more uniform distribution pattern is obtained for the $1.625 \mathrm{~mm}$ thickness, fine grain structure case compared to the $0.5 \mathrm{~mm}$ thickness, coarse grain structure case.

Average absolute value of strain at the bend area through the thickness
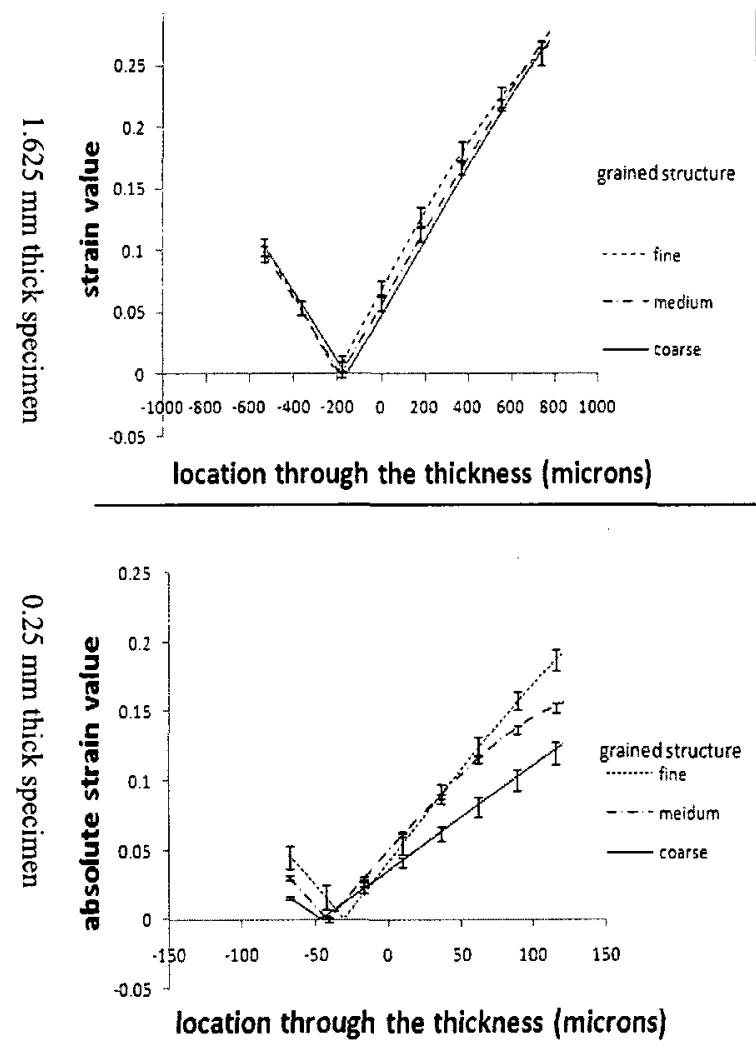

Average hardness profile through the thickness
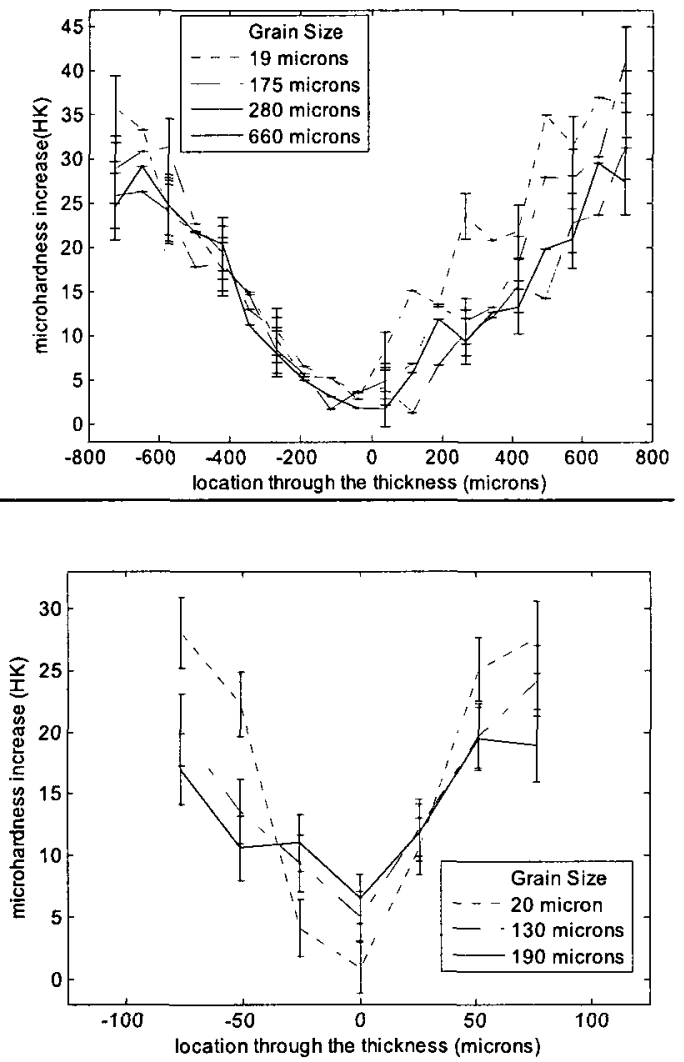

Figure 3-9 Strain distribution results obtained from DIC analysis were compared to the previous results obtained by hardness measurement in Parasiz et al. [11] for $1.625 \mathrm{~mm}$ and $0.25 \mathrm{~mm}$ thickness specimens with various grained structures. 
Average absolute value of strain at the bend area through the thickness
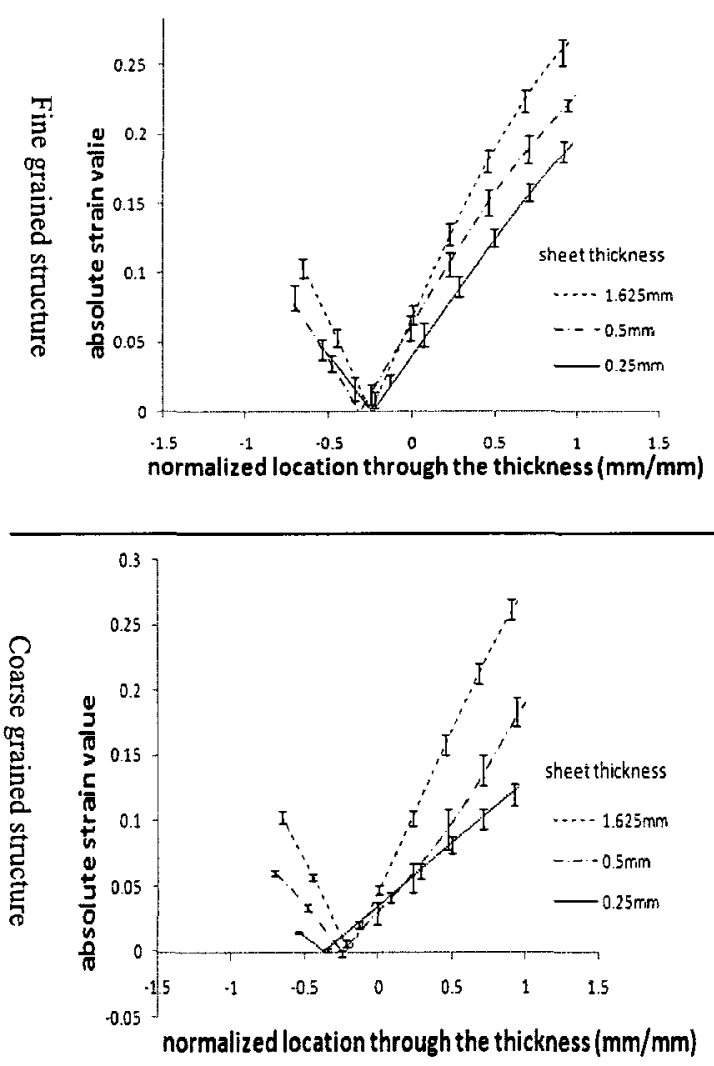

Average hardness profile through the thickness
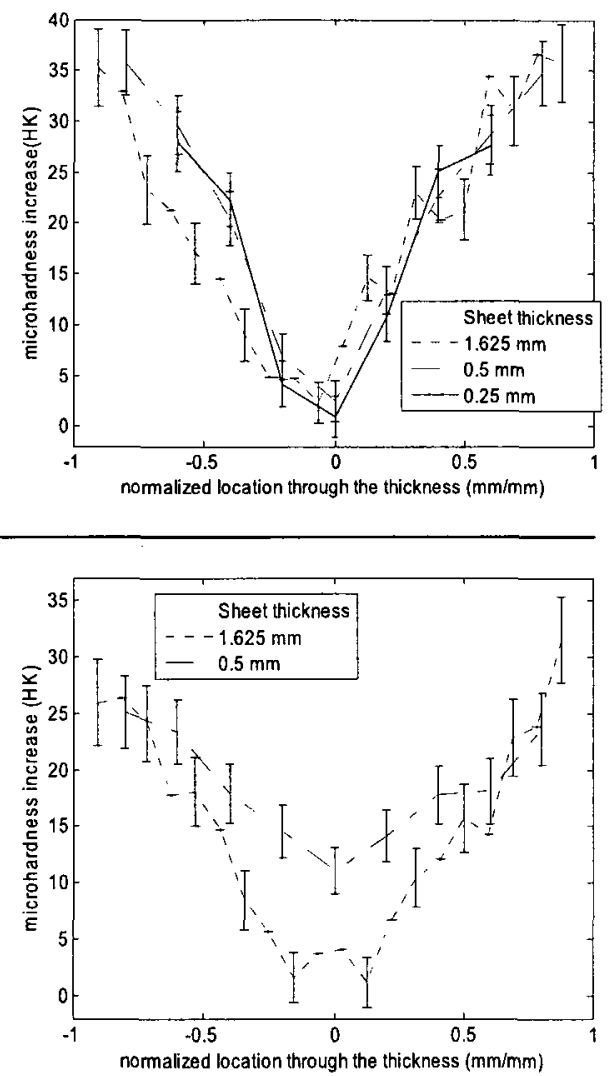

Figure 3-10 Strain distribution results obtained from DIC analysis were compared to the previous results obtained by hardness measurement in Parasiz et al. [11] for specimens with fine and coarse grained structures 


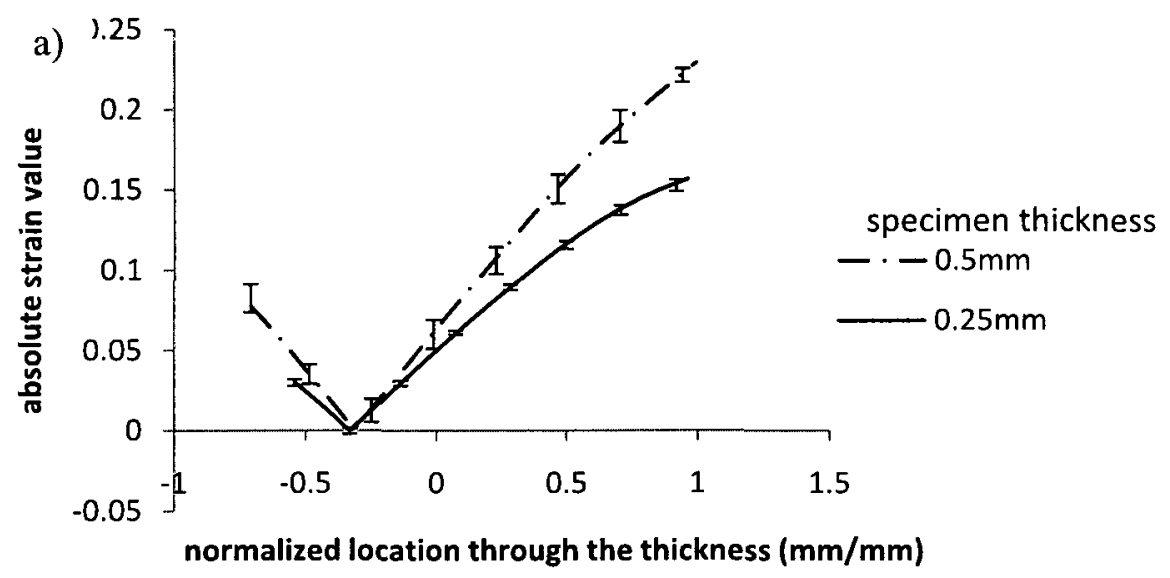

b)
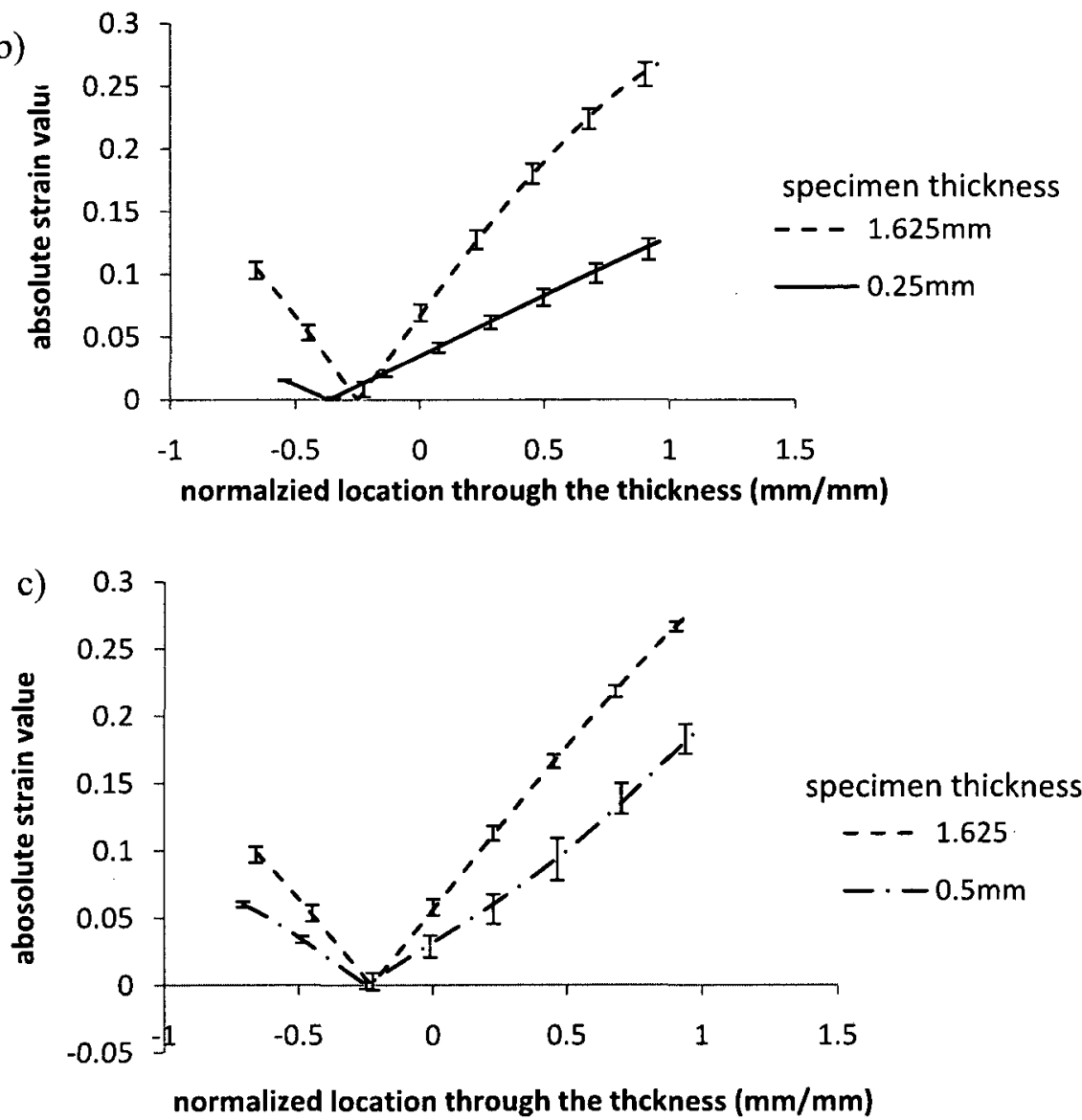

Figure 3-11 Stain distribution of a) $20 \mu \mathrm{m}$, b) $65 \mu \mathrm{m}$ and c) $150 \mu \mathrm{m}$ with various specimen sizes. 
By scaling the process by the law of similarity, a strain of $21.4 \%$ should theoretically be achieved on the outer surface for all specimens. As the specimen size decreases, the strain value changes over a shorter distance; thus, the strain gradients imposed by the deformation geometry should theoretically become steeper. However, the measured strain gradients from the DIC did not show this trend, see Fig. 3-5. Also the strain values at the surface for the smaller specimens were less than predicted, see Fig. 3-13. Perhaps this effect only occurs at the side surface and a plane strain condition does not exist there. During deformation, coarse grains distribute the deformation more evenly especially for smaller thickness specimens. Thus, for larger grain sizes, the penetration of plastic deformation from the surface regions to the inner region occurs during bending, and less plastic deformation (i.e., lower strain values) are presented at the outer surfaces.

a)

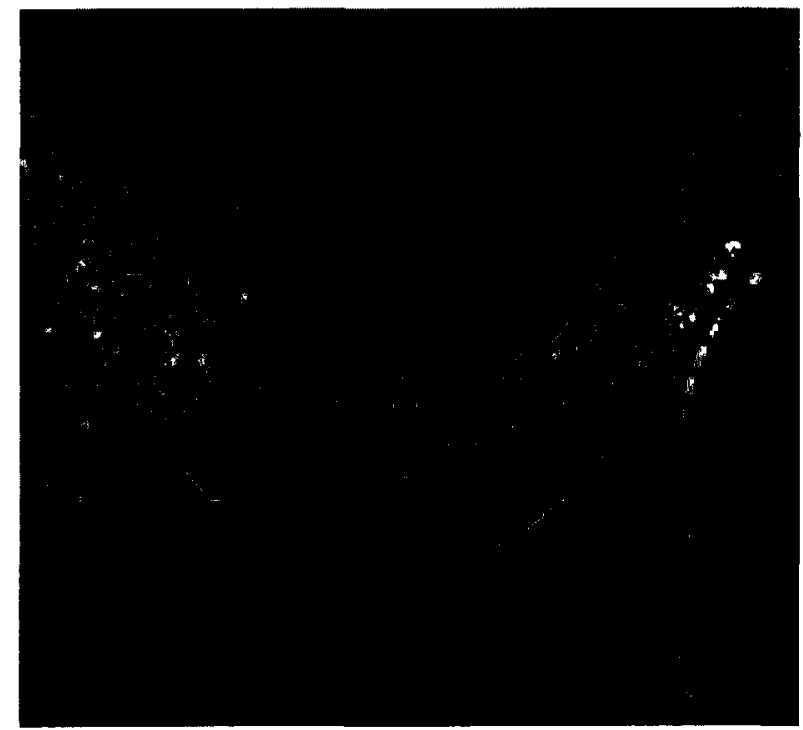


b)

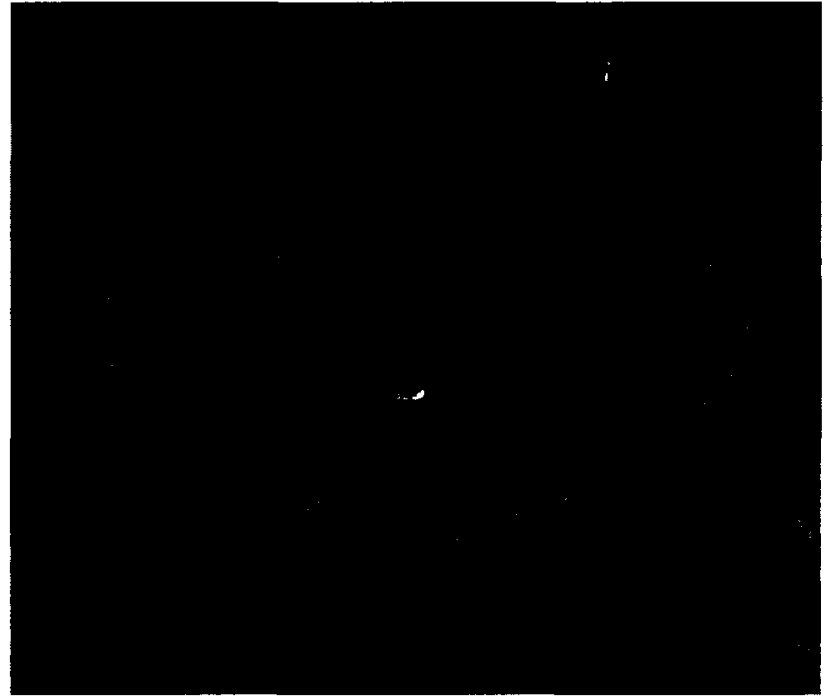

Figure 3-12: Strain distribution data for a) $1.625 \mathrm{~mm}$ thickness specimen with fine grain structure, b) $0.5 \mathrm{~mm}$ thickness specimen with coarse grain structure.

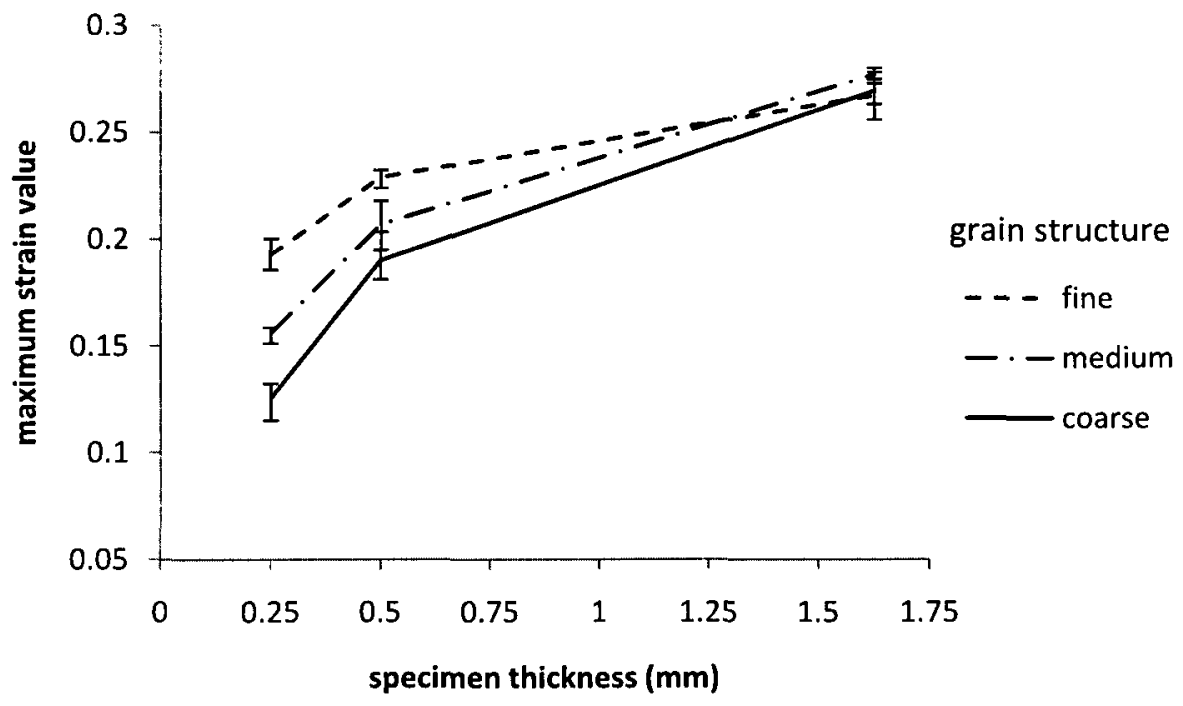

Figure 3-13: Maximum strain on tension surface for various specimen thicknesses and grain structures. 


\section{INVESTIGATION OF STRAIN GRADIENT HARDENING DURING MICROBENDING}

\subsection{Background}

As components with proportional feature and tooling sizes are miniaturized, strain gradients through the cross-section theoretically should increase. This causes strain gradient hardening as the density of geometrically necessary dislocations increases. This will lead to higher required forces in the process than expected. However, based on our experimental results presented in Chapter 3, these strain gradients may not increase as predicted and may even decrease. In this chapter, an analytical model to predict the dislocation density increases, and thus strain gradient hardening based on theoretical increases in the strain gradients during microbending, is presented. These results match previous research reasonably well in terms of the feature size where modest and significant strain gradient hardening was observed. But further consideration of the observed strain gradients with miniaturization will have to be considered.

\subsection{Introduction}

Strain hardening of crystalline metals at the microscale is significantly affected by the 
mode of the deformation. Ashby in 1970 introduced the distinction between statistically stored dislocations, SSDs, and geometrically necessary dislocations, GNDs [18]. While the former are generated generally during homogeneous deformation, the latter are generated due to the plastic shear strain gradients during inhomogeneous deformation.

For the simplest one dimensional case, the density of GND, $\rho_{\mathrm{GND}}$, can be calculated according to plastic shear strain gradients $(\chi)$ as [19]:

$$
\rho_{\mathrm{GND}}=\frac{1}{\mathrm{~b}}\left(\frac{\mathrm{d} \gamma_{\mathrm{pl}}}{\mathrm{dy}}\right)=\frac{\chi}{\mathrm{b}}
$$

where $b$ is the Burgers vector and $d \gamma_{p l}$ is the plastic shear strain. The GNDs contribute to strain hardening of materials. Ashby proposed a Taylor-type relation to estimate their contribution on strain hardening:

$$
\tau=\mathrm{cGb} \sqrt{\rho_{\mathrm{SSD}}+\rho_{\mathrm{GND}}}
$$

where $\mathrm{G}$ is the shear modulus and $\mathrm{c}$ is a numerical factor, typically on the order of $\mathrm{c} \sim$ $0.2-0.4$ and $\rho_{S S D}$ is the density of statistically stored dislocations.

The contribution of GNDs has been used to explain the increased strain hardening of very small metallic specimens when they undergo inhomogeneous deformation, such as in torsion of thin wires [20], bending of thin sheets [12] or nanoindentation of a surface [21]. For example, during torsion, strain gradients are generated through the diameter of wires. When the specimen diameter size is scaled down to the order of tens of microns, the strain gradients become steep. As a result the density of GNDs increases significantly 
and the specimen experiences strain gradient hardening (SGH). From Fig 4-1 the strength of the wires increased by a factor of 3 when reducing the diameter from $170 \mu \mathrm{m}$ to 12 $\mu \mathrm{m}$. In addition, the SGH becomes significant when the specimen feature size is on the order of tens of microns. Similarly, as the specimen size approaches the grain size in plane-strain microbending, the penetration of deformation occurs for coarse grained cylinders thus producing increased hardening in the middle of the sheet material [11].

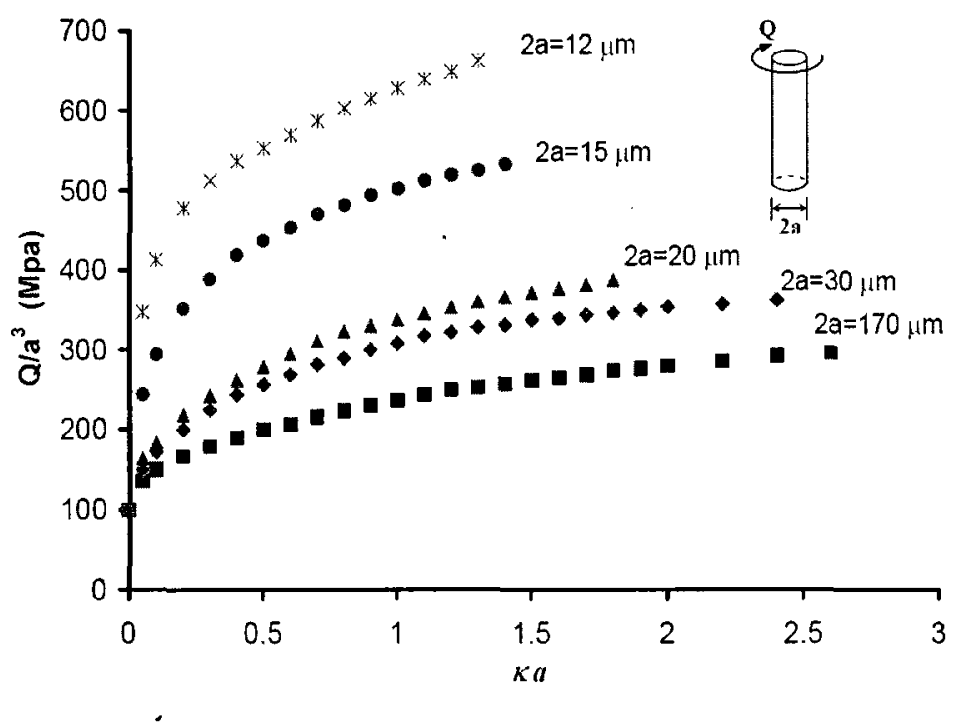

Figure 4-1: Dependence of strength on specimen size during microtorsion of thin copper wires [20].

In this chapter, an analytical model is presented to calculate the GND density during a bending process. This formulation is then used for a specific case to show the effect of initial sheet thickness on GND density. These results are compared to force-displacement data from Chapter 3 and results from Stolken and Evans [12]. Similar results are observed for the thickness where modest and significant increases in strain gradient 
hardening occur.

\subsection{Analytical model}

\subsubsection{Geometric and Strain Relationships}

As described in Chapter 1, during pure bending, a moment is applied and produces tension and a length increase on the outer surface of the bend and compression and a length decrease on the inner surface of the bend. The radius of the bend, $r$, is to the central axis of the sheet thickness. See Fig. 4-2 for a schematic of this pure bending process.
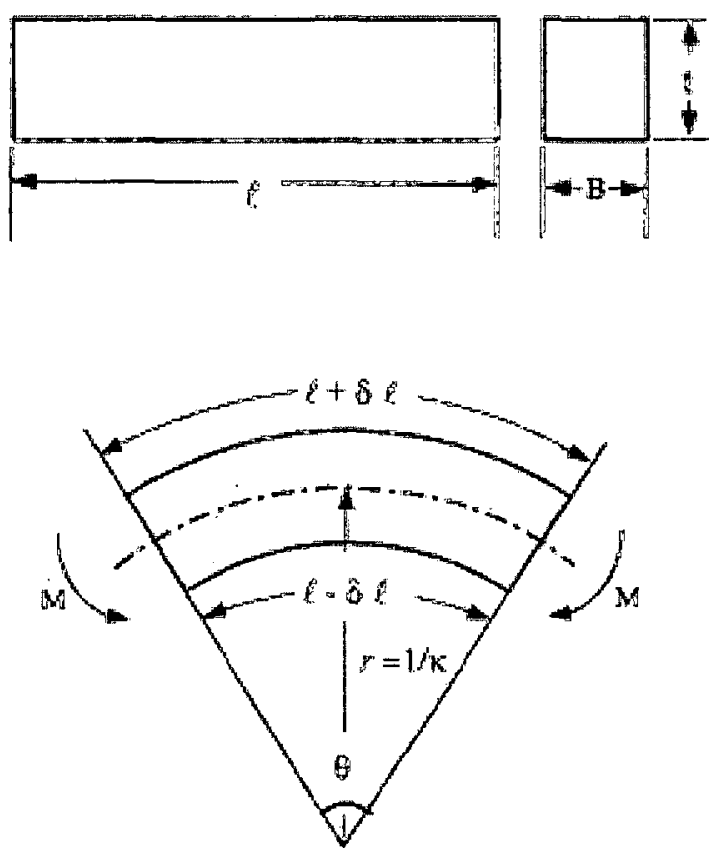

Figure 4-2. Geometric parameters for pure bending. 
The length of the central axis $\ell$, does not change in the process and is defined by:

$$
\ell=r \theta
$$

The change in length can be calculated by subtracting the length of the outer surface from that of the inner surface:

$$
\delta \ell=\frac{t \theta}{2}
$$

The strain gradient $(\eta)$ through the cross-section is assumed to be linear and can be defined using the strain on the outer surface and the strain on the inner surface by substituting in Eqs. (4.3) and (4.4):

$$
\eta=\frac{2 \delta \ell}{t \ell}=\frac{2}{t} \frac{t \theta}{2 \ell}=\frac{\theta}{\ell}=\frac{\theta}{r \theta}=\frac{1}{r}=\kappa
$$

where $\kappa$ is the radius of curvature. This is a well-known derivation that can be found in several references $[22,23]$ but is provided here for clarification. Note that the coefficient of friction was excluded in this model.

\subsubsection{The Density of GND During Microbending}

The GND density $\left(\rho_{G N D}\right)$ can be defined as [24]:

$$
\rho_{G N D}=\frac{\eta}{b}=\frac{1}{r b}=\frac{\kappa}{b}
$$

where $b$ is the burgers vector for the material. Since the final radius in this case is 1.5 times the sheet thickness, a relation between the density of GND and sheet thickness can 
be generated from:

$$
\rho_{G N D}=\frac{2}{3 t b}
$$

\subsection{Analytical Model Results}

Equation (4.7) was used to calculate the density of GND for 3-point bending experiments described in Chapter 2 on CuZn30 with varying sheet thicknesses and a punch radius that is 1.5 times the thickness of the sheet. See Fig. 4-3. The burgers vector for the material was assumed to be that of pure copper (i.e., $254 \mathrm{~nm}$ ). Note that this figure is applicable for the instant when the specimen has been fully bent around the punch radius.

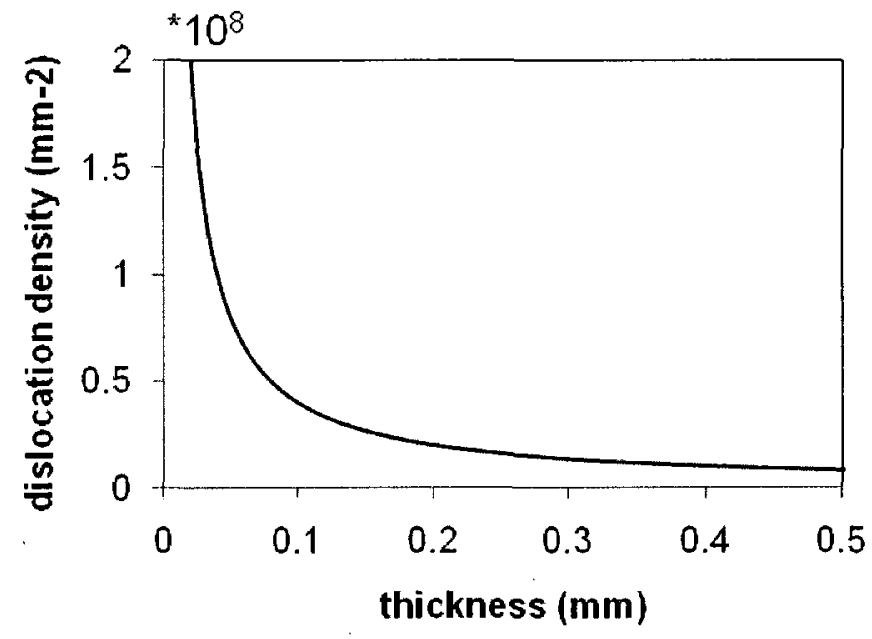

Figure 4-3: Dislocation density increase versus specimen thickness (t) for CuZn30 and a radius of curvature of $1.5 \mathrm{t}$.

Significant strain gradient hardening from GND density increases was not predicted 
based on these results until the sheet thickness was less than approximately $0.03 \mathrm{~mm}$. However, some modest increase in the GND density was predicted when decreasing the specimen thickness, see Fig 4-4 (which was also presented in Fig 3-4). That is, the increased bending force with miniaturization is assumed to have occurred due to increases in the dislocation density with miniaturization.

Fig. 4-5 from Stolken and Evans [12] shows how the non-dimensional bending moment varied as the sheet thickness was reduced assuming a length scale of $5.3 \mu \mathrm{m}$. While Figs. 4-3 and 4-5 are for different parameters, i.e., GND density and non-dimensional bending moment, these variables are related to the SGH of the material. Furthermore, process parameters such as radius of curvature and the material are not the same between these two studies. However, the approximate thickness value where significant SGH occurs is approximately the same, i.e., when the thickness is less than $0.03 \mathrm{~mm}$. Thus, the analytical model presented here and the one in [12] provide reasonably consistent results.

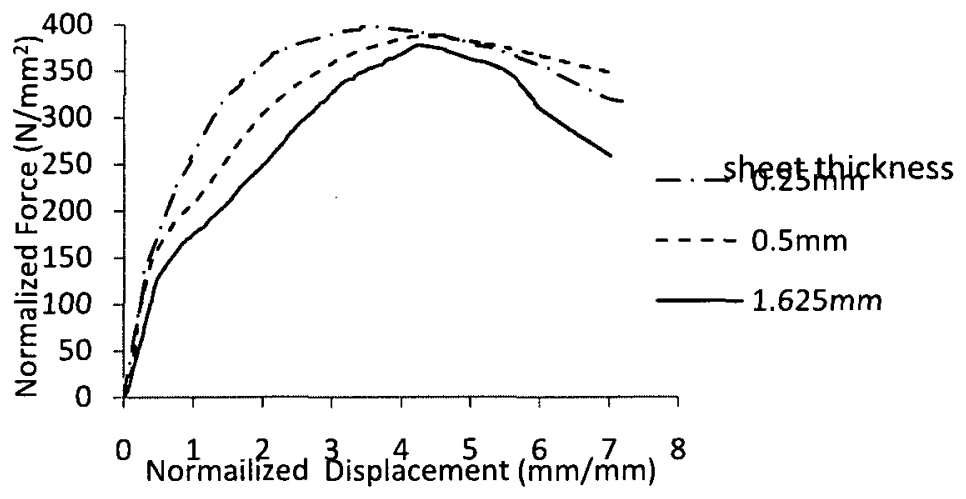

Figure 4-4: Normalized punch force for 3-point bending experiments with $\mathrm{CuZn} 30$ and fine grain structure. 


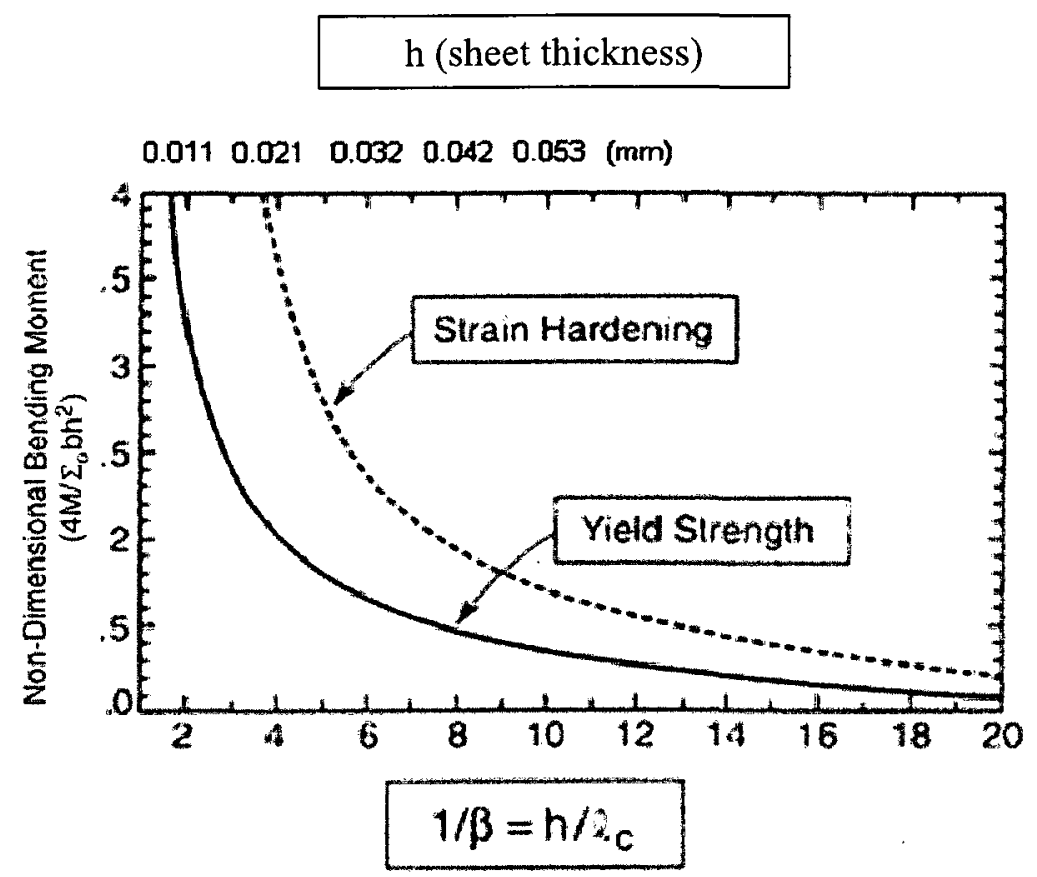

Figure 4-5: Data from Stolken and Evans [14] with an assumed length scale of $5.3 \mu \mathrm{m}$. 


\section{CHAPTER 5}

\section{CONCLUSIONS}

In this research, the dependence of deformation in microbending on specimen size and grain size was investigated via the Digital Image Correlation method. It was found that the strain distribution values through the thickness are affected by the grain size when the specimen size is miniaturized. For the largest size $1.625 \mathrm{~mm}$ thickness specimens, the strain distributions are similar for the various grain size sheets. However, for $0.5 \mathrm{~mm}$ and $0.25 \mathrm{~mm}$ thickness specimens, the strain gradients were less steep as the grain size increased. This is a result of penetration of plastic deformation to the inner regions during microbending of the coarse grained structures. These results were consistent with past research which used microhardness measurements to characterize the deformation size effects; however, note that these are two distinct deformation parameters so the comparison was limited to the trends observed. Another finding was that the strain at the surface of the smaller sized specimens was significantly less than expected based on the geometry of the process. Images of the various specimen sizes did not show any differences in the deformation. Thus, further experiments are required to investigate this effect. Finally, results showed that the neutral axis location (as a percent of the thickness) increased with miniaturization of the specimen size, except for the fine grained structure which was relatively constant. The rationale for this finding is that penetration of deformation, in particular from the tensile surface, causes the increased shifting of the neutral axis. Alternatively, the neutral axis location decreased as the grain size increased, 
except for the $0.25 \mathrm{~mm}$ cases. The rationale for this finding is that coarse grains are limited in their deformation, thus decreasing the neutral axis shifting. Again, further experiments are required to investigate this effect as well.

In addition, an analytical model was presented to calculate the Geometrically Necessary Dislocation (GND) density during a bending process. This formulation was then used for a specific case to show the effect of initial sheet thickness on GND density. It was found that the GND density increases with a decrease in final specimen thickness. The result from Stolken and Evans [12] and the force-displacement data from the microbending experiments were compared with analytical model results and similar trends were observed.

However, from the microbending experimental results, the strain gradient slope decreases as the specimen size decreases and/or the grain size increases with miniaturization despite the increased peak bending force observed for the fine grain case, see Fig. 3-6 a). This less steep strain gradient result conflicts with the analytical model assumption that the strain gradient increases. This may be due to the strain being measured at the side surface of the specimen and a plane strain condition not existing at this location. Thus, further research is needed to study and verify this phenomenon. 


\section{CHAPTER 6}

\section{FUTURE WORK}

The analytical model presented in Chapter 4 suggests that significant strain gradient hardening from GND density increases will not occur until the sheet thickness is less than approximately $0.03 \mathrm{~mm}$. Past research has also shown that deformation size effects exist with miniaturization and thus the strain distribution through the cross-section varies compared to the analytical model results. In addition, from the microbending experiment results, the strain gradients decrease as the specimen size decreased and/or the grain size increased with miniaturization. This result conflicts with the analytical model presented in Chapter 4. Thus, more tests need to be conducted to measure the strain on the bottom surface of the specimen to see if the reduced strain measured with DIC system is only a side surface effect. Also, measurements of springback and strain after unloading should be investigated. Finally, more experimental tests using smaller size specimens are needed in order to further analyze this effect and validate the model. In particular, additional experiments with specimens that are $<0.1 \mathrm{~mm}$ are required.

An experimental set-up which uses Digital Imaging Correlation was presented. Future experiments will be conducted with this experimental set-up and modifications to the analytical model to produce accurate results will be investigated. The DIC method provides a straight forward means to obtain the strain distribution from the experiments. 
Correlated Solutions also provides a microscope system to use with their software, VIC Micro. The maximum magnification for the microscope is $11.5 \mathrm{x}$ which should allow analysis of specimens with a thickness of $<0.1 \mathrm{~mm}$ and thus better assess the strain distribution with miniaturization. Note that the difficulty with using this system will be producing a speckled pattern for thicknesses $<0.1 \mathrm{~mm}$. Then in order to characterize the SGH behavior, nanohardness, instead of microhardness, measurements will be obtained thus eliminating the indentation size to thickness size concern.

Furthermore, the DIC system will be use to investigate different microforming processes, such as Electrical-Assisted Forming processes of microbending and microscale compression [24] tests with corresponding size effects. This may help to provide a fundamental understanding of this phenomenon. 


\section{REFERENCES}

[1] Component Engineering Inc, http://www.componenteng.com/

[2] Cao, J., Krishnan, N., Wang, Z., Lu, H., Liu, W.K., Swanson, A. (2004) "Microforming -Experimental investigation of the extrusion process for micropins and its numerical simulation using RKEM", ASME Journal of Manufacturing Science and Engineering, Vol. 126, pp. 642-652.

[3] Cavaliere, P., Gabrielli, F., Fratini, L., "Bending of Very Thin Sheets: the Influence of Thickness on Material Characterisation and Elastic Springback", Proceedings of the 8th International Conference on Metal Forming, Krakow (Pl), 2000, pp. 405-410.

[4] Diehl, A., Engel, U., Geiger, M., "Investigation of the Spring-back behaviour in metal foil forming", Proceedings of the $24^{\text {th }}$ IDDRG-Conference, Besancon, Frankreich, 2005.

[5] Kocanda, A., Prejs, T., "The effect of miniaturization on the final geometry of the bend products", Metal Forming 2000, Pietrzyk et al. (eds), 2000 Balkema, Rotterdam, pp. $375-378$.

[6] Geiger, M., Messner, A., Engel, U., Kals, R., and Vollersten, F., 1995, "Design of Microforming Processes-Fundamentals, Material Data and Friction Behavior,'Proc. of 9th International Cold Forging Congress, Solihull, UK, May, pp. 155-163.

[7] Raulea, L. V., Govaert, L. E., and Baaijens, F. P. T., 1999, "Grain and Specimen Size Effects in Processing Metal Sheets," Advanced Technology of Plasticity, Proc. of 6th ICTP, Sept. 19-24, 2, pp. 939-944.

[8] Hill, R., 1950, "The Mathematical Theory of Plasticity", Oxford, London, pp.288-294.

[9] Lacono, C., Sinke, J., Benedictus, R., " Prediction of Minimum Bending Ratio of Aluminum Sheets From Tensile Material Properties", Journal of Manufacturing Science and Engineering", Vol. 132, April 2010, pp. 11-19.

[10] Kals, T. A., and Eckstein, R., 2000, "Miniaturization in Sheet Metal Working," J. Mater. Process. Technol., 103, pp. 95-101.

[11] Parasiz, S., VanBenthysen, R., and Kinsey, B.L., "Deformation Size Effects Due to Specimen and Grain Size in Microbending", Journal of Manufacturing Science and Engineering, Vol. 32, in press. 
[12] Stolken, J.S., Evans, A.G., "A Microbend Test Method for Measuring the Plasticity Length Scale", Acta Mater., Vol.14, No. 14, 1998, pp.5109-5115.

[13] 2008, ASTM Standards E1 12-96, Section 3, Metals Test Methods and Analytical Procedures, Vol. 03.01, ASTM, Philadelphia, pp. 290-315.

[14] T. C. Chu, W. F. Ranson, M. A. Sutton, and W. H. Peters, "Application of digitalimage-correlation techniques to experimental mechanics", Exp. Mech. 25, 1985, pp. 232-244.

[15] Speckle Pattern Fundamentals, www.correlated solutions.com.

[16] Kalpakjian, S., and Schmid, S. R., 2006, Manufacturing Engineering and Technology, Prentice-Hall, Englewood Cliffs, NJ, p. 445.

[17] Richard M. Onyancha and Brad L. Kinsey "Experimental Investigation of Microbending Process", Transactions of the North American Manufacturing Research Institute of SME, Vol. 36, 2008, pp. 65-71

[18] Ashby, M.F., "The Deformation of Plastically Non-homogeneous Materials," Philosophical Magazine, Vol. 21 .,1970, pp. 399-424.

[19] Mughrabi, H., "On the Current Understanding of Strain Gradient Plasticity", Mat.Sci. and Eng. A, Vol. 387-389, 2004, pp.209-213.

[20] Fleck, N.A., Muller, G. M., Ashby, M.F. and Hutchinson, W., "Strain Gradient Plasticity: Theory and Experiment", Acta Metall. Mater., Vol. 42, No. 2, 1994, pp.475-487.

[21] Nix, W.D. and Gao, H., "Indentation Size Effects in Crystalline Materials: A Law For Strain Gradient Plasticity”, J. Mech. Phys. Solids, Vol. 46, No. 3, 1998, pp. 411 425.

[22] Soboyejo. W. O., "Mechanical Properties of Engineered Materials", Marcel Dekker, NY, pp 210-212.1

[23] Courtney, T.H., “Mechanical Behavior of Materials”, Mcgraw-Hill Inc, IL, 2000

[24] Gao, H., Huang, Y., Nix, W.D., and Hutchinson, J.W., "Mechanism Based Strain Gradient Plasticity: Theory", J. Mech. Phys. Solids, Vol. 47, 1999, pp. 1239-1263.

[25] Christine M. Dzialo, Michael S. Siopis, Klaus J. Winemann, and Brad L. Kinsey "Effect of Current Density and Zinc Content during Electrical-Assisted Forming of Copper Alloys", accepted to the Annals of CIRP, 2010. 
APPENDICIES 


\begin{abstract}
APPENDIX A
The strain analysis procedure of DIC system is presented below, and this is taken from Vic-2D Manual provided by www.correlated solutions.com.

\section{Loading Images}

The first step to obtain displacement field is to load the images to analyze into the program. Vic-2D distinguishes between the Reference Image and Deformed Images. The reference image is the one taken of the specimen when no load was applied, can be selected by clicking on the button on the tool bar. The deformed images is the one taken during loading of the specimen.
\end{abstract}

\title{
Choosing the Subset and Step Size
}

Before or after selecting an area of the reference image for correlation, the subset and step size have to be selected. The subset size controls the area of the image that is used to track the displacement between images. The subset size has to be large enough to ensure that there is a sufficiently distinctive pattern contained in the area used for correlation. The step size controls the spacing of the points that are analyzed during correlation. If a step size of 1 is chosen, a correlation analysis is performed at every pixel inside the areaof-interest. A subset size of 2 means that a correlation will be carried out at every other pixel in both the horizontal and vertical direction, etc. 


\section{Selecting an Area-of-Interest}

To select an area-of-interest of the reference image to use for analysis, select one of the AOI tool buttons from the tool bar. After selecting the desired type of AOI, click on the reference image to select the actual area.

Vic-2D currently supports the following types of AOIs:

- Extensometer: Two points specified by the end-points of a line.

- Line: A number of points evenly spaced along a line.

- Rectangle: Points spaced on an even grid contained in a rectangular area.

- Polygon: Points spaced on an even grid contained in a polygon.

To specify a particular type of AOI, select the corresponding entry in the Edit menu or the appropriate button on the tool bar. The selected AOI type will be indicated by the state of the corresponding tool button (sunken for active).

After selecting the AOI type, move the cursor to the desired position in the reference window and click the left mouse button. User can now move the mouse to the next position, e.g. the end of the line or the second corner of the rectangle. Clicking the left mouse button again will complete the AOI selection for all AOI types except polygons. For polygon selection, a double-click is used to specify the last point of the polygon.

During AOI selection, a dashed yellow line is drawn to indicate the outline of the selection. 


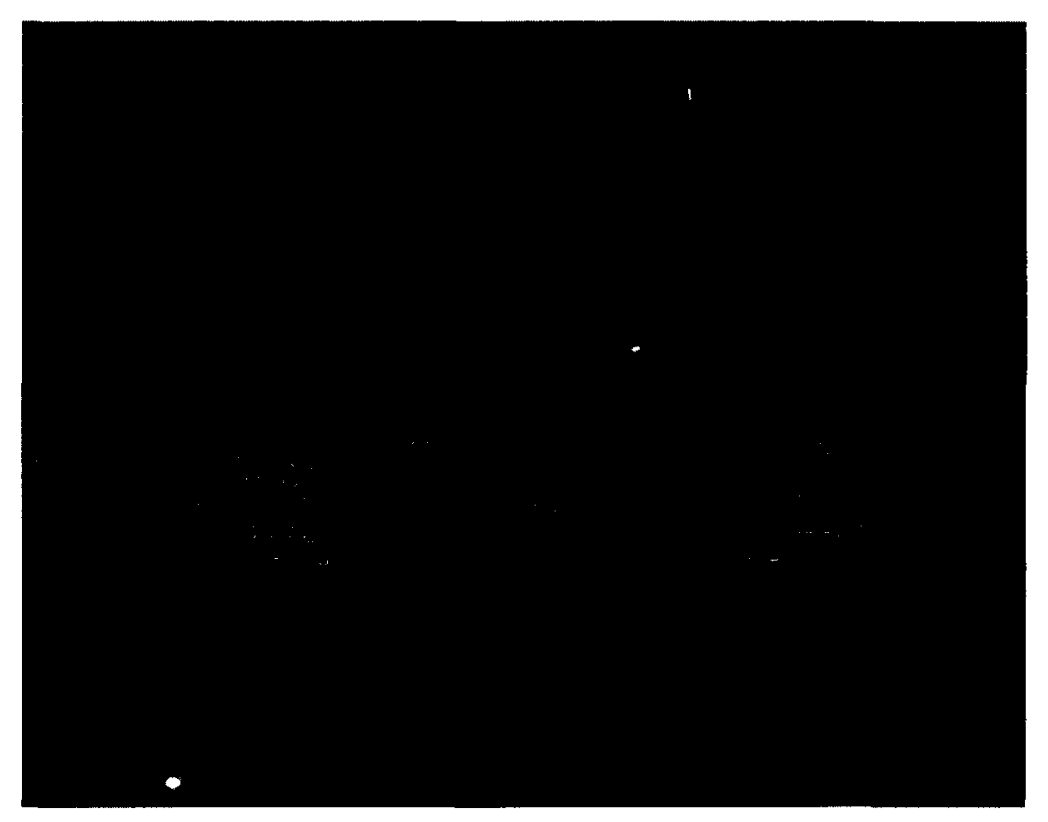

Figure A-1: Selecting an area of interest.

\section{The Seed Point}

The term Seed Point is used to refer to the point in the reference image where the correlation is started. The correlation algorithms use the results from the seed point to obtain an initial guess for the second point analyzed and continue in this manner until all points in the AOI are analyzed.

For rectangular and polygon AOIs, the seed point can be moved to a user-specified location in the AOI. If a rectangle or polygon $\mathrm{AOI}$ is the currently active $\mathrm{AOI}$ in the reference image, the Move Seed entry in the Edit menu or the tool button on the tool bar can be activated. A new seed point can now be selected by clicking on a point inside the 
currently active polygon AOI. The placement of the seed point can greatly influence the amount of work required to select an initial guess. Ideally, the seed point should be placed in the area of the image that underwent the smallest amount of motion during the test.

\section{$\underline{\text { Guess Selection }}$}

If the motion between successive images is larger than a few pixels, the image correlation algorithm requires an initial guess. This initial guess can be provided to the algorithm using the initial guess dialog.

The placement of the seed point can facilitate or eliminate the need for providing an initial guess. Generally, it is best to place the seed point of the AOI in the area of the image that underwent the least amount of motion during the test. For instance, if a specimen is tested in a tensile frame, the seed point should be placed as close to the stationary grip as possible. In many cases, good placement of the seed point eliminates the need to give an initial guess at all, since the algorithms can cope with small motion increments. If there is no stationary point or point with small motion in the image, an initial guess has to be provided. To make this process easier, it is recommended to place the seed point on an easily recognizable feature in the image. The area surrounding the seed point in the reference image is displayed in the box labeled Reference AOI. Next to it, the pattern in the deformed image is displayed in the box labeled Deformed AOI. The image in the bottom left of the dialog shows the deformed image. The listbox on the bottom right can be used to select the deformed image for which the initial guess is being 
provided.

To select corresponding points in the reference and deformed image, the red cross in the bottom left image has to be placed in the area of the deformed image that corresponds to the area around the seed point in the reference image. This can be done by clicking and moving the mouse in the bottom left image. The image in the Deformed AOI box is updated whenever a new point is selected in the deformed image. Once the Reference AOI and Deformed AOI images an initial guess can be specified for the translation components of the displacement only, or for the complete deformation including the partial derivatives of the displacement. The latter will only be necessary in cases where the strains and/or rotations are very high.

Follow the steps below to specify a translation guess:

- Select a common point in the Reference AOI and Deformed AOI by clicking the mouse.

- Click the Add Point button.

- Optionally repeat the first two steps for more corresponding points.

- Click the Calculate button.

- The calculated displacements will be shown. Click Accept.

- The icon in the file list will change to a green check to indicate that an initial guess has been selected for the current image.

- Repeat this procedure for all deformed files shown in the file list. 


\section{Running the data analysis and view results}

To run the correlation analysis, click the button on the tool bar. During data analysis, the program will display information on the progress of the analysis. After the images have been processed, a list of data files will be displayed in the list view on the left side of the program window. A contour plot of the displacement data can be opened by doubleclicking on a data-file in list. By right-clicking on the plot, user can select which variable to display from the context-menu.

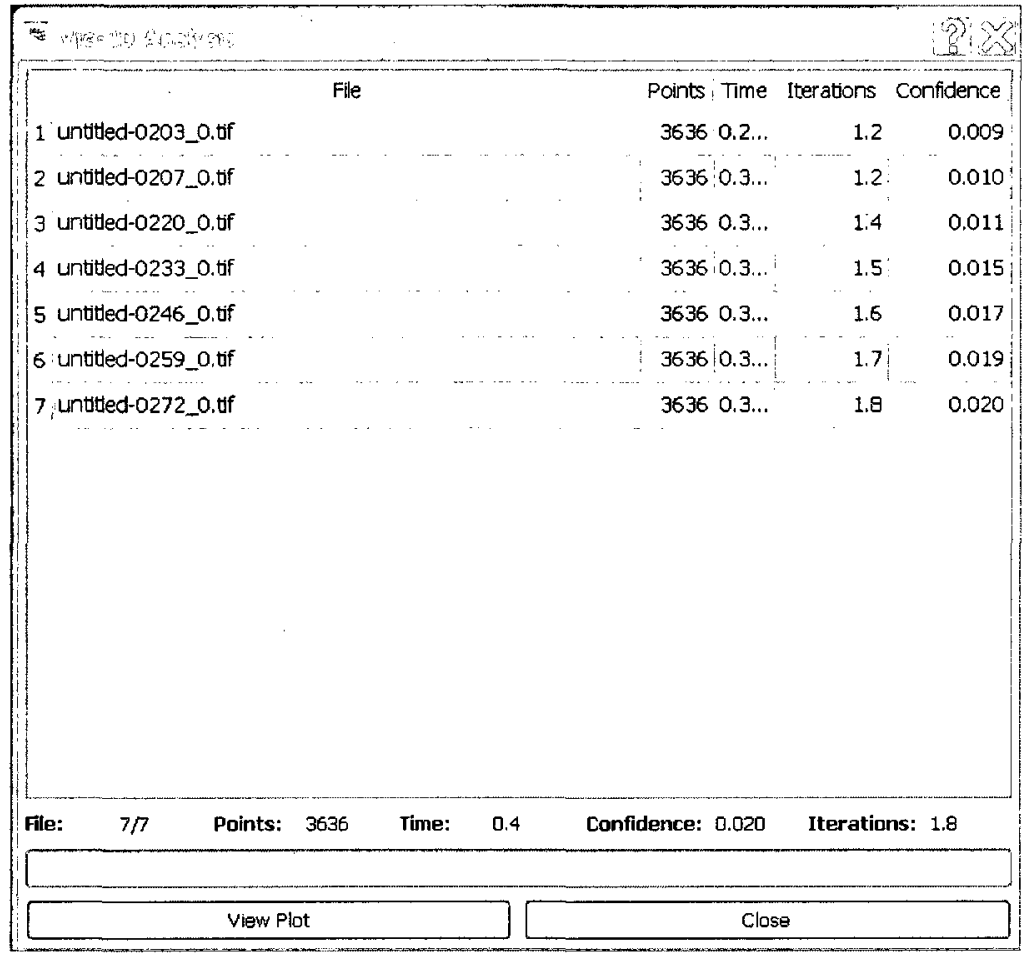

Figure A-2: Image analysis result window.

\section{Calculating Strains}


The algorithm used to calculate the strains computes the partial derivatives of the displacement field from a first-order polynomial approximation of the displacement in a local neighborhood. The strains are calculated from the partial derivatives of the displacement using the Lagrange strain tensor equations. Once the displacement fields have been calculated from the speckle image, the strains can be calculated by selecting the Calculate Strain entry on the Data menu.

\section{Contour Plots}

A contour plot of a data file can be displayed by right-clicking on a data file in the list on the left of the workspace and selecting Contour Plot from the menu that appears. Alternatively, a file can be selected from the Data-Contour Plot menu. An example of a contour plot is displayed below. The data file for the plot can be selected from the dropdown menu in the top-left corner. The contour variable to display can be selected from the drop-down menu on the left of the plot. The contour range can be manually adjusted by entering the minimum and maximum value in the input fields on the left. Click Apply to apply the new range.

If the auto-rescaling checkbox is checked, the range is automatically calculated whenever a new data file is displayed. To turn off auto-rescaling, uncheck the checkbox. This is useful for making animations. The opacity of the contour plot can be adjusted to better identify features in the underlying image so that data can easily be extracted at known 
locations. The inspector tools allow to probe data points, extract data along a line and extract sequence data for points or circular areas.

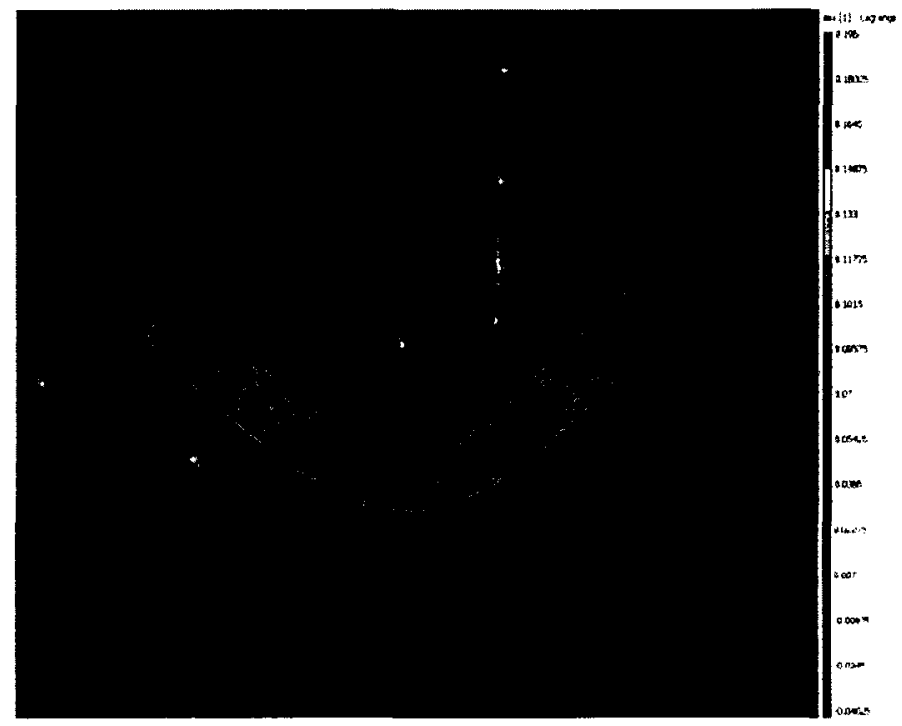

Figure A-3: Contour plot of strain distribution data.

\section{Extracting Data}

After an item has been added to the plot or selected using the select tool, the Extract button becomes active. By pressing the Extract button, either sequence data or line data can be extracted from the data file(s). Contour plots can be saved as PNG or BMP images. Press the Save Plot button on the bottom left of the window to save the current contour plot. Note: You can also copy the plot and paste it into other programs. Simply select the Copy 
from the menu by right-clicking anywhere on the plot. Contour plots can be animated by clicking on the Animation button in the bottom left corner of the window. 


\section{APPENDIX B}

This MATLAB program creats a density of GND model (chapter 4).

$\operatorname{PSSD}=\left((1.094)^{\wedge} 2\right)^{*}\left(10^{\wedge} 8\right)$

$b=0.254 *\left(10^{\wedge}-6\right)$

$\mathrm{r}=0.9:-0.01: 0$;

$\mathrm{PGND}=(3937007.87) . / \mathrm{r}$

$\mathrm{t}=\mathrm{r} / 2$

$\operatorname{plot}\left([0,8],\left[\left((1.094)^{\wedge} 2\right)^{*}\left(10^{\wedge} 8\right),\left((1.094)^{\wedge} 2\right)^{*}\left(10^{\wedge} 8\right)\right]\right)$

hold on;

$\operatorname{plot}(r, P G N D, ' k ')$;

legend('PSSD','PGND');

$\operatorname{axis}\left(\left[\begin{array}{lll}0 & \left.\left.0.5-\inf 2 * 10^{\wedge} 8\right]\right) ;\end{array}\right.\right.$

xlabel('thickness of thin metal sheet(mm)');

ylabel('Dislocation Density $\left.\left(\mathrm{mm}^{\wedge}-2\right)^{\prime}\right)$;

$\mathrm{t} 1=$ input('Please enter the thickness of sample:');

$\mathrm{s} 1=1.5 * \mathrm{t} 1$

$\operatorname{PSSD} 1=\left((1.2182)^{\wedge} 2\right)^{*}\left(10^{\wedge} 8\right)$

$\mathrm{rl}=6:-0.01: \mathrm{s} 1$

PGND1=(3937007.87)./r1;

$\operatorname{plot}\left([0,8],\left[\left((1.2182)^{\wedge} 2\right)^{*}\left(10^{\wedge} 8\right),\left((1.2182)^{\wedge} 2\right)^{*}\left(10^{\wedge} 8\right)\right]\right)$

hold on;

plot(r1,PGND1,'g');

legend('PSSD1','PGND1'); 
xlabel('radius of the curvature before springback $(\mathrm{mm})$ ');

ylabel('Dislocation Density $\left.\left(\mathrm{mm}^{\wedge}-2\right)^{\prime}\right)$;

s2=input('Enter the radius after springback (mm):')

$\mathrm{r} 2=6:-0.1: \mathrm{s} 2$

$\operatorname{PSSD} 2=\left((1.2182)^{\wedge} 2\right)^{*}\left(10^{\wedge} 8\right)$

PGND2 $=(3937007.87) . / \mathrm{r}$;

$\operatorname{plot}\left([0,8],\left[\left((1.2182)^{\wedge} 2\right)^{*}\left(10^{\wedge} 8\right),\left((1.2182)^{\wedge} 2\right)^{*}\left(10^{\wedge} 8\right)\right]\right)$

hold on;

plot(r2,PGND2,'g');

legend('PGND2','PSSD2')

xlabel('radius of the curvature after springback $(\mathrm{mm})$ ');

ylabel('Dislocation Density $\left.\left(\mathrm{mm}^{\wedge}-2\right)^{\prime}\right)$; 